\title{
SPECTRA OF TWO-DIMENSIONAL MODELS FOR THIN PLATES WITH SHARP EDGES*
}

\author{
A. CAMPBELL ${ }^{\dagger}$, S. A. NAZAROV ${ }^{\ddagger}$, AND G. H. SWEERS ${ }^{\S}$
}

\begin{abstract}
We investigate the spectrum of the two-dimensional model for a thin plate with a sharp edge. The model yields an elliptic $3 \times 3$ Agmon-Douglis-Nirenberg system on a planar domain with coefficients degenerating at the boundary. We prove that in the case of a degeneration rate $\alpha<2$, the spectrum is discrete, but, for $\alpha \geq 2$, there appears a nontrivial essential spectrum. A first result for the degenerating scalar fourth order plate equation is due to Mikhlin. We also study the positive definiteness of the quadratic energy form and the necessity to impose stable boundary conditions. These results differ from the ones that Mikhlin published.
\end{abstract}

Key words. thin plate, sharp edge, varying thickness, essential spectrum, stable boundary conditions

AMS subject classifications. 74K20, 35J58, 35P99

DOI. $10.1137 / 100788719$

1. Introduction. The model system for a thin plate with a sharp edge has some peculiar features. The most remarkable one is that the spectrum for this elliptic boundary will contain a nonempty essential part if the edge is sharp enough. This result is in striking contrast to model for a plate with uniform thickness or a less sharp edge. The appearance of an essential spectrum provokes wave behavior in finite elastic bodies with cuspidal surfaces. This phenomenon is of practical use in modern engineering when constructing filters and dampers of elastic waves (see [12, 20] for specific engineering devices known as "vibrating black holes"). A first place where the behavior of thin plates of nonuniform thickness is studied is the book of Mikhlin. For a good understanding of the problem, it is fundamental to recall some of his results for the plate problem.

1.1. Recalling Mikhlin's results on plates with sharp edges. In this section we formulate some results described in the second Russian edition [19] of the classical book by Mikhlin on variational methods. In the first edition, which is available in an English translation [18], this part is not presented.

Let $\omega \subset \mathbb{R}^{2}$ be the longitudinal cross section of a thin isotropic plate with variable thickness. We assume that $\omega$ is a domain with a $C^{2}$-boundary $\partial \omega$, and we write $\bar{\omega}=\omega \cup \partial \omega$ for the compact closure. The thickness function $h$ is smooth inside $\omega$, is positive in $\omega$, but vanishes on $\partial \omega$. More precisely, setting

$$
\rho(x)=\operatorname{dist}(x, \partial \omega) \text { for } x \in \bar{\omega} .
$$

we may define the system of local curvilinear coordinates $(n, s)$ in

$$
\omega_{\ell}=\{x \in \omega ; \operatorname{dist}(x, \partial \omega)<\ell\}
$$

\footnotetext{
* Received by the editors March 15, 2010; accepted for publication (in revised form) September 21, 2010; published electronically December 7, 2010.

http://www.siam.org/journals/sima/42-6/78871.html

${ }^{\dagger}$ Laboratoire de Mathématiques Nicolas Oresme, BP 5186 Université de Caen, F-14032 Caen, France (alain.campbell@unicaen.fr).

${ }^{\ddagger}$ Institute of Problems of Mechanical Engineering, Russian Academy of Sciences, V. O., Bolshoi prosp., 61, St. Petersburg, Russia (srgnazarov@yahoo.co.uk).

$\S$ Mathematisches Institut, Cologne University, Germany (gsweers@math.uni-koeln.de), and DIAM, Delft University of Technology, Delft, The Netherlands.
} 
for $\ell>0$ sufficiently small, such that for some $\alpha>0$ we have

$$
h(x)=n^{\alpha}(H(s)+\tilde{h}(x)) \text { for } x \in \omega_{\ell} .
$$

Here $n=\rho(x)$ is the oriented distance to $\partial \omega$, and $s$ is the arc length along $\partial \omega$. Furthermore, $H$ is a smooth positive function on $\partial \omega$, and $\tilde{h}$ is a smooth function on $\bar{\omega}$ with $\tilde{h}=0$ on $\partial \omega$.

According to [16] (see also [19, sect. 37, pp. 201-206]) the differential equation for the deflection $w$ of the plate reads

$$
L\left(x, \nabla_{x}\right) w(x)=f(x) \text { for } x \in \omega,
$$

where $f$ is the density of the transversal load and

$$
\begin{aligned}
& L\left(x, \nabla_{x}\right)=D\left(\frac{\partial^{2}}{\partial x_{1}^{2}} h(x)^{3} \frac{\partial^{2}}{\partial x_{1}^{2}}+\frac{\partial^{2}}{\partial x_{2}^{2}} h(x)^{3} \frac{\partial^{2}}{\partial x_{2}^{2}}\right. \\
& \left.+\nu\left(\frac{\partial^{2}}{\partial x_{1}^{2}} h(x)^{3} \frac{\partial^{2}}{\partial x_{2}^{2}}+\frac{\partial^{2}}{\partial x_{2}^{2}} h(x)^{3} \frac{\partial^{2}}{\partial x_{1}^{2}}\right)+2(1-\nu) \frac{\partial^{2}}{\partial x_{1} \partial x_{2}} h(x)^{3} \frac{\partial^{2}}{\partial x_{1} \partial x_{2}}\right) .
\end{aligned}
$$

The constant $D=\frac{E}{12\left(1-\nu^{2}\right)}$ is the reduced cylindrical rigidity of the plate material with the Young modulo $E$ and the Poisson ratio $\nu \in\left[0, \frac{1}{2}\right]$.

The elastic energy $\mathcal{E}():. C_{c}^{\infty}(\omega) \rightarrow \mathbb{R}$ is defined by $\mathcal{E}(w)=\frac{1}{2} a_{e}(w, w)$ with

$$
\begin{aligned}
a_{e}(w, w):=D \int_{\omega} h(x)^{3}( & \left|\frac{\partial^{2} w}{\partial x_{1}^{2}}(x)\right|^{2}+\left|\frac{\partial^{2} w}{\partial x_{2}^{2}}(x)\right|^{2} \\
& \left.+2 \nu \frac{\partial^{2} w}{\partial x_{1}^{2}}(x) \frac{\partial^{2} w}{\partial x_{2}^{2}}(x)+2(1-\nu)\left|\frac{\partial^{2} w}{\partial x_{1} \partial x_{2}}(x)\right|^{2}\right) d x .
\end{aligned}
$$

One finds

$$
a_{e}(w, w) \geq D(1-\nu) \sum_{j, k=1}^{2} \int_{\omega} h(x)^{3}\left|\frac{\partial^{2} w}{\partial x_{j} \partial x_{k}}(x)\right|^{2} d x .
$$

For bounded $\omega$, Poincaré's inequality shows that $a_{e}(w, w)=0$ implies $w=0$ and hence that $w \mapsto a_{e}(w, w)^{\frac{1}{2}}$ is a norm on $C_{c}^{\infty}(\omega)$. Here $C_{c}^{\infty}(\omega)$ is the space of infinitely differentiable functions which are compactly supported in the bounded domain $\omega$. We will define the function space $\mathfrak{H}$ as the completion of $C_{c}^{\infty}(\omega)$ with respect to this norm $w \mapsto a_{e}(w, w)^{\frac{1}{2}}$. The elastic energy will have a natural extension $\mathcal{E}():. \mathfrak{H} \rightarrow \mathbb{R}$.

For the plate with a transversal load that is clamped at the edge, we consider the functional corresponding to the potential energy, that is, the elastic energy minus the work of external forces,

$$
\mathcal{J}(w):=\mathcal{E}(w)-\int_{\omega} w(x) f(x) d x
$$

A solution should minimize $\mathcal{J}$ :

$$
w=\operatorname{argmin}\{\mathcal{J}(\tilde{w}) ; \tilde{w} \in \mathfrak{H}\} .
$$

The following conclusions, related to the degenerating rate $\alpha$ in (1.2), can be found in Mikhlin's book [19, sect. 37]:

Copyright (c) by SIAM. Unauthorized reproduction of this article is prohibited. 
- if $\alpha<\frac{1}{3}$, elements in $\mathfrak{H}$ and their first-order derivatives keep zero trace on $\partial \omega$, i.e., the differential equation (1.3) must by supplied with the full set of boundary conditions,

$$
w(x)=0, \quad \nabla_{x} w(x)=0 \quad \text { for all } x \in \omega
$$

- if $\frac{1}{3} \leq \alpha<1$, elements in $\mathfrak{H}$ themselves keep zero trace on $\partial \omega$ and the differential equation (1.3) needs one but only one boundary condition,

$$
w(x)=0 \quad \text { for all } x \in \omega ;
$$

- if $\alpha \geq 1$, elements in $\mathfrak{H}$ do not keep zero trace on $\partial \omega$ and the differential equation (1.3) cannot by supplied with a boundary condition.

Remark 1.1. A miscalculation in [19] led to the threshold $\frac{2}{3}$ in the last two assertions. Here we use the corrected number, namely 1 . The error occurs in a nonexplicit computation between formulas (6) and (7) on page 203, where it is written: "A simple analysis demonstrates that...."

For $\frac{1}{3} \leq \alpha<1$, the above result means that any function in $C^{2}(\bar{\omega})$ that vanishes on $\partial \omega$ admits a sequence $\left\{w_{k}\right\}_{k \in \mathbb{N}} \subset C_{c}^{\infty}(\omega)$, such that

$$
a_{e}\left(w-w_{k}, w-w_{k}\right) \rightarrow 0 \text { for } k \rightarrow \infty .
$$

In view of (1.6), the quadratic form (1.5) degenerates only on the space $\mathfrak{R}$ of rigid motions:

$$
\mathfrak{R}=\left\{w: w(x)=a_{0}+a_{1} x_{1}+a_{2} x_{2}, a_{i} \in \mathbb{R}\right\} .
$$

Since no nontrivial rigid motion verifies the condition (1.9), the quadratic form $a_{e}$ stays positive definite in the space $\mathfrak{H}$.

For $\alpha \geq 1$, however, any function $w \in C^{2}(\bar{\omega})$ can be approximated by $\left\{w_{k}\right\}_{k \in \mathbb{N}} \subset$ $C_{c}^{\infty}(\omega)$ in the sense of (1.10) so that the completion procedure may fail to determine a Hilbert space $\mathfrak{H}$. Indeed, as shown in [19], the form (1.5) is positive definite on $\mathfrak{H}$ only in the case $\alpha<\alpha_{0}$ with again an erroneously calculated $\alpha_{0}=\frac{3}{2}$. The correct $\alpha_{0}$ equals 1.

To counteract this effect of the degeneration at the boundary we introduce the quadratic form

$$
a(w, w)=a_{e}(w, w)+a_{k}(w, w),
$$

with

$$
a_{k}(w, w)=\gamma \int_{\omega} h(x)|w(x)|^{2} d x .
$$

for $\gamma>0$ some constant. The additional term (1.13) in the quadratic form in (1.12) can be viewed as a kinetic term due to a vibration $e^{i \nu t} w(x)$. It leads us to the spectral problem

$$
\left\{\begin{aligned}
& L\left(x, \nabla_{x}\right) w(x)= \lambda \gamma h(x) w(x) \text { for } x \in \omega, \\
& w \in \mathfrak{H}
\end{aligned}\right.
$$

where $\gamma$ is a material constant and $\lambda$ stands for the spectral parameter. 

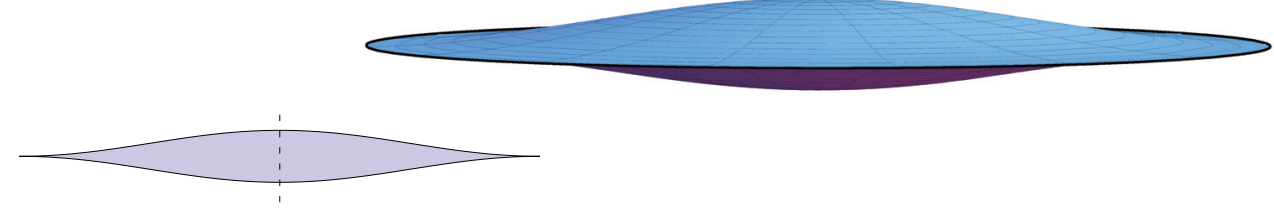

FIG. 1. On the right, a plate with a sharp edge, and a cross section on the left.

The form (1.12) is surely positive definite on any function space and, therefore, the completion of $C_{c}^{\infty}(\omega)$ in the norm $w \mapsto a(w, w)^{\frac{1}{2}}$ provides a Hilbert space for which we still write $\mathfrak{H}$. The above assumptions for the boundary conditions are still valid.

The weak formulation (see [14]) of the spectral problem (1.14) with the appropriate set of boundary conditions reads as follows.

Problem 1.2. Find $\lambda \in C$ and $w \in \mathfrak{H}$, with $w \neq 0$, such that the integral identity

$$
a_{e}(w, \psi)=\lambda a_{k}(w, \psi) \quad \text { for all } \psi \in \mathfrak{H},
$$

is valid, where $a_{k}(w, w)$ as in (1.13).

We shall give a suitable formulation of the spectral problem (1.15) in section 3 . Here we only mention that, under the assumption

$$
\alpha<2 \text {, }
$$

it is proved in [19] that the spectrum of the problem is fully discrete.

The aim of this paper is to extend the above-mentioned results to anisotropic plates and, moreover, to show that in the case $\alpha \geq 2$ problem (1.14) gets a nonempty essential spectrum. Note that in the literature several definitions of essential spectrum appear. However, in a self-adjoint setting, as we will have here, all definitions coincide.

1.2. The general formulation of the plate problem. To shorten formulas, we write the system in matrix form (see, e.g., [15, 28]),

$$
L\left(x, \nabla_{x}\right) u(x)=\mathcal{D}\left(-\nabla_{x}\right)^{\top} \mathcal{A}(x) \mathcal{D}\left(\nabla_{x}\right) u(x)=f(x) \text { for } x \in \omega,
$$

where $u=\left(v_{1}, v_{2}, w\right)^{\top}$ is the displacement vector regarded as a column, $w=u_{3}$ is the deflection, and $v_{j}$ the displacement in the direction $x_{j}, j=1,2$. Here $\top$ stands for transposition and $\mathcal{D}$ is the $6 \times 3$-matrix of differential operators,

$$
\mathcal{D}\left(\nabla_{x}\right)^{\top}=\left(\begin{array}{cccccc}
\partial_{1} & 0 & 2^{-\frac{1}{2}} \partial_{2} & 0 & 0 & 0 \\
0 & \partial_{2} & 2^{-\frac{1}{2}} \partial_{1} & 0 & 0 & 0 \\
0 & 0 & 0 & 2^{-\frac{1}{2}} \partial_{1}^{2} & 2^{-\frac{1}{2}} \partial_{2}^{2} & \partial_{1} \partial_{2}
\end{array}\right),
$$

where $\partial_{j}=\frac{\partial}{\partial x_{j}}$. The stiffness matrix $\mathcal{A}(x)$ of size $6 \times 6$ takes the form

$$
\mathcal{A}(x)=\int_{-h_{-}(x)}^{h_{+}(x)}\left(\begin{array}{cc}
A(x, \zeta) & -\sqrt{2} \zeta A(x, \zeta) \\
-\sqrt{2} \zeta A(x, \zeta) & 2 \zeta^{2} A(x, \zeta)
\end{array}\right) d \zeta
$$

while $A(x, \zeta)$ is a positive definite $3 \times 3$-matrix constructed from the elastic moduli of a heterogeneous anisotropic material filling the three-dimensional (3d) plate

$$
\Omega=\left\{(x, \zeta): x \in \omega \text { and }-h_{-}(x)<\zeta<h_{+}(x)\right\} .
$$

An example of a plate with a sharp edge is shown in Figure 1. 
Note that the transversal variable $\zeta$ is stretched such that the originally thin plate is characterized after rescaling by

$$
\sup _{y \in \omega} h(y)=1, \quad h(y)=h_{+}(y)+h_{-}(y) \text { for } y \in \omega .
$$

Representation formulas of the type in (1.19) were derived under different assumptions in $[38,21],[28$, sect. 4,2 , pp. 179-189] and others and we accept them without a specification of the matrix $A$, although the rigorous dimension reduction from 3 -d to two-dimensional (2-d) has not been done yet in the case of a plate with the sharp edge, i.e., in the case

$$
h_{ \pm}(x)=n^{\alpha}\left(H_{ \pm}(s)+\tilde{h}_{ \pm}(x)\right)
$$

with the same notation as in (1.2), $H=H_{+}+H_{-}$, and $\tilde{h}=\tilde{h}_{+}+\tilde{h}_{-}$, and $H_{ \pm}$and $\tilde{h}_{ \pm}$are smooth nonnegative functions with $\tilde{h}_{ \pm}=0$ on $\partial \omega$. We may assume that

$$
\left|h_{ \pm}(x) / h(x)\right| \leq c_{h} .
$$

For our analysis, we need only that the matrix function $A$ is smooth, symmetric, and positive definite in the set $\bar{\Omega}$. Then formula (1.19) ensures that the $3 \times 3$-blocks of the matrix

$$
\mathcal{A}(x)=\left(\begin{array}{ll}
\mathcal{A}_{\sharp \sharp}(x) & \mathcal{A}_{\sharp 3}(x) \\
\mathcal{A}_{3 \sharp}(x) & \mathcal{A}_{33}(x)
\end{array}\right)
$$

satisfy the inequalities

$$
\begin{aligned}
& c_{\sharp} \rho(x)^{\alpha}\left|\xi_{\sharp}\right|^{2} \leq \xi_{\sharp}^{\top} \mathcal{A}_{\sharp \sharp}(x) \xi_{\sharp} \leq C_{\sharp} \rho(x)^{\alpha}\left|\xi_{\sharp}\right|^{2} \text { for all } \xi_{\sharp} \in \mathbb{R}^{3}, \\
& c_{3} \rho(x)^{3 \alpha}\left|\xi_{3}\right|^{2} \leq \xi_{3}^{\top} \mathcal{A}_{33}(x) \xi_{3} \leq C_{3} \rho(x)^{3 \alpha} \quad\left|\xi_{3}\right|^{2} \text { for all } \xi_{3} \in \mathbb{R}^{3},
\end{aligned}
$$

where $c_{\sigma}, C_{\sigma}$ are positive constants. Moreover, elements of the block $\mathcal{A}_{3 \sharp}(x)=$ $\mathcal{A}_{\sharp 3}(x)^{\top}$ are bounded by $C \rho(x)^{2 \alpha}$.

If the elastic material is homogeneous, which means $A$ is constant, then

$$
\mathcal{A}(x)=\left(\begin{array}{cc}
h(x) A & -\frac{1}{\sqrt{2}} h(x)\left(h_{+}(x)-h_{-}(x)\right) A \\
-\frac{1}{\sqrt{2}} h(x)\left(h_{+}(x)-h_{-}(x)\right) A & \frac{2}{3} h(x)\left(h_{+}(x)^{2}-h_{+}(x) h_{-}(x)+h_{-}(x)^{2}\right) A
\end{array}\right),
$$

so that the geometrical symmetry $\left(h_{+}=h_{-}=\frac{1}{2} h\right)$ leads to the block-diagonal matrix

$$
\mathcal{A}(x)=\left(\begin{array}{cc}
h(x) A & \mathbf{0}_{3} \\
\mathbf{0}_{3} & \frac{1}{6} h(x)^{3} A
\end{array}\right)
$$

where $\mathbf{0}_{N}$ is the null matrix of size $N \times N$. Then the structure of (1.18) and (1.27) results in a splitting of the system of differential equations (1.17) into a fourth order equation for the deflection $w$ and a system of second order equations for the vector $v=\left(v_{1}, v_{2}\right)$ of the longitudinal displacements. Moreover, in the case of a isotropic material we have

$$
A=\left(\begin{array}{ccc}
\frac{E}{1-\nu^{2}} & \frac{E \nu}{1-\nu^{2}} & 0 \\
\frac{E}{1-\nu^{2}} & \frac{E}{1-\nu^{2}} & 0 \\
0 & 0 & \frac{E}{1+\nu}
\end{array}\right)
$$

Copyright (c) by SIAM. Unauthorized reproduction of this article is prohibited. 
(see, e.g., [28, sect. 4,2]) and the above mentioned fourth order equation takes the form (1.3) with the differential operator (1.4).

We also outline that an asymptotic analysis of the spectral elasticity problem in a thin plate (see, for example, $[5,8]$ for the isotropic and [26], [28, Chap. 6] for the anisotropic and inhomogeneous case) leads to the system of differential equations

$$
L\left(x, \nabla_{x}\right) w(x)=\lambda \gamma(x) \mathbf{e}_{3} w(x) \quad \text { for } x \in \omega,
$$

where $\mathbf{e}_{3}=(0,0,1)^{\top}$ and $\gamma$ is a smooth positive function in $\omega$, namely

$$
\gamma(x)=n^{\alpha}(\Gamma(s)+\tilde{\gamma}(x)) \quad \text { for } x \in \omega \cap \mathcal{V},
$$

with $\Gamma$ and $\tilde{\gamma}$ being smooth in $\partial \omega$ and $\bar{\omega}$, respectively, and satisfying $\Gamma>0$ and $\tilde{\gamma}=0$ on $\partial \omega$. The distinguishing feature of system (1.29) is just the presence of the spectral parameter $\lambda$ in the third equation only.

The system (1.29) describes transversal oscillations of the plate, while the asymmetry of physical and/or geometric characteristics, that is, $\mathcal{A}_{\sharp 3} \neq \mathbf{0}_{3}$, stimulates longitudinal vibrations as well. The pure longitudinal oscillations of the plate (see $[8,26]$ and $[28$, Chap. 6]) are described by the following system of two differential equations:

$$
\mathcal{D}_{\sharp}\left(-\nabla_{x}\right)^{\top} \mathcal{A}_{\sharp \sharp}(x) \mathcal{D}_{\sharp}\left(\nabla_{x}\right) v(x)=\mu \gamma(x) v(x) \quad \text { for } x \in \omega,
$$

where $\mu$ is a spectral parameter, $\mathcal{A}_{\sharp \sharp}$ is a $3 \times 3$-block in $(1.24)$ and $\mathcal{D}_{\sharp}\left(\nabla_{x}\right)^{\top}$ is the upper left-hand $2 \times 3$-block of the matrix $\mathcal{D}\left(\nabla_{x}\right)^{\top}$ in (1.18).

1.3. The structure of this paper. In section 2 we give, based on several variants of the one-dimensional Hardy's inequality, an equivalent norm in the function space $\mathfrak{H}=\mathfrak{H}_{\sharp} \times \mathfrak{H}_{3}$ that is suitable for the variational formulation of problems (1.17) and (1.29). The distribution of the weights in the norm allows for a conclusion on the existence of traces on $\partial \omega$ and the validity of the boundary conditions (1.8) or (1.9). As a direct consequence we obtain Theorem 2.10 on the existence and uniqueness of a solution to problem (1.17) together with the description of the kernel and the cokernel in the case of nonuniqueness.

In section 3 we investigate spectral problems. First, we observe that the spectrum of problem (1.31) on $\mathfrak{H}_{\sharp}$ is always discrete. Moreover, when $\alpha \in(0,1)$ the spectrum consists of strictly positive eigenvalues and when $\alpha \geq 1, \mu=0$ is an eigenvalue of multiplicity 3 . We then reprove Mikhlin's result on the discrete spectrum of problem (1.15) (pure bending) under condition (1.16). We also extend the result for problem (1.29), where the interaction of deflection and longitudinal deformation occurs. We finish the section by constructing the singular Weyl sequence in the case $\alpha \geq 2$, which shows that, according to the Weyl criterion (cf. [36, sect. 133], [2, Thm. 9.1.2]), the essential spectrum of the problem is not empty. We remark that in order to apply the most general results of the theory of self-adjoint operators in Hilbert spaces, one needs a reduction of the system to a scalar integro-differential equation (cf. [27], [28, Chap. 7]).

In section 4 we make some speculations on related open questions such as the asymptotic analysis of thin 3-d plates with sharp edges, the structure of the essential spectrum, and the formulation of appropriate radiation conditions at the edge. 


\section{Main results concerning solvability.}

2.1. Some variants of Hardy's inequality. We list some nonstandard variations on the well-known Hardy type inequalities (cf. [10]).

Lemma 2.1. Let $U \in C^{1}([0, \ell])$.

- If $U(0)=0$ and $\beta<0$, or if $U(\ell)=0$ and $\beta>0$, then

$$
\int_{0}^{\ell} t^{2 \beta-1} U(t)^{2} d t \leq \frac{1}{\beta^{2}} \int_{0}^{\ell} t^{2 \beta+1} U^{\prime}(t)^{2} d t
$$

- If $U(0)=0$, then

$$
\int_{0}^{\ell} t^{-3} \ln \left(\frac{2 \ell}{t}\right)^{-2} U(t)^{2} d t \leq 64 \int_{0}^{\ell} t^{-1} \ln \left(\frac{2 \ell}{t}\right)^{-2} U^{\prime}(t)^{2} d t .
$$

- If $U(\ell)=0$, then

$$
\begin{gathered}
\int_{0}^{\ell} t^{-1} \ln \left(\frac{2 \ell}{t}\right)^{-2} U(t)^{2} d t \leq 4 \int_{0}^{\ell} t U^{\prime}(t)^{2} d t \\
\int_{0}^{\ell} t^{-1} \ln \left(\frac{2 \ell}{t}\right)^{-4} U(t)^{2} d t \leq \frac{4}{9} \int_{0}^{\ell} t \ln \left(\frac{2 \ell}{t}\right)^{-2} U^{\prime}(t)^{2} d t .
\end{gathered}
$$

Remark 2.2. The function

$$
t \mapsto \ln \left(\frac{2 \ell}{t}\right)^{-1}:[0, \ell] \rightarrow \mathbb{R}
$$

is zero at zero but this zero is of "order" less than any positive power $\alpha$ of $t \mapsto t^{\alpha}$. See Figure 2. By the way, we write $\ln \left(\frac{2 \ell}{t}\right)^{-1}:=\left(\ln \left(\frac{2 \ell}{t}\right)\right)^{-1}$ etc.

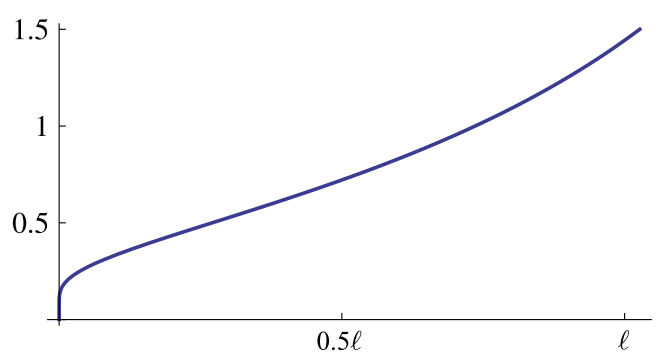

FIG. 2. The graph of $y=\ln \left(\frac{2 \ell}{x}\right)^{-1}$.

Since Hardy published the inequality named after him in 1920, many generalizations have been obtained. The books of Kufner with Opic [34] and with Persson [13] supply us with a wide range of Hardy-type inequalities with weights. For example, for (2.1) see [34, Ex. 6.9]. The estimates that contain a logarithmic term also follow from the general result of [34] but to check the conditions is rather involved.

Proof. For the reader's convenience we will prove one of these estimates, namely, (2.2). Similar as for the classical version, one starts with the Newton-Leibniz formula $2 \int_{a}^{b} U(t) U^{\prime}(t) d t=\left[U(t)^{2}\right]_{a}^{b}$, integrates this identity, next changes the order of 
both integrations, uses a sufficiently sharp elementary function estimate, and applies Cauchy-Schwarz to find $X \leq c \sqrt{X Y}$ and concludes $X \leq c^{2} Y$. For the case (2.2) this proceeds as follows.

One may directly check that

$$
t \mapsto(4 \ell-3 t) t^{-1} \ln \left(\frac{2 \ell}{t}\right)^{-2}
$$

is a strictly decreasing function on $(0, \ell)$. Then we have for $\ell \geq t>\tau \geq 0$ that

$$
t^{-1} \ln \left(\frac{2 \ell}{t}\right)^{-2} \leq \frac{4 \ell-3 t}{\ell} t^{-1} \ln \left(\frac{2 \ell}{t}\right)^{-2}<\frac{4 \ell-3 \tau}{\ell} \tau^{-1} \ln \left(\frac{2 \ell}{\tau}\right)^{-2} \leq 4 \tau^{-1} \ln \left(\frac{2 \ell}{\tau}\right)^{-2},
$$

and we find the following estimates:

$$
\int_{\tau}^{\ell} t^{-3} \ln \left(\frac{2 \ell}{t}\right)^{-2} d t \leq 4 \tau^{-1} \ln \left(\frac{2 \ell}{\tau}\right)^{-2} \int_{\tau}^{\ell} t^{-2} d t \leq 4 \tau^{-2} \ln \left(\frac{2 \ell}{\tau}\right)^{-2} .
$$

Next we find

$$
\begin{gathered}
\int_{0}^{\ell} t^{-3} \ln \left(\frac{2 \ell}{t}\right)^{-2} U(t)^{2} d t=2 \int_{0}^{\ell} \int_{t=\tau}^{\ell} t^{-3} \ln \left(\frac{2 \ell}{t}\right)^{-2} \\
U^{\prime}(\tau) U(\tau) d t d \tau \leq 8 \int_{0}^{\ell}\left|U^{\prime}(\tau)\right||U(\tau)| \tau^{-2} \ln \left(\frac{2 \ell}{\tau}\right)^{-2} d \tau \\
\leq 8\left(\int_{0}^{\ell} \tau^{-3} \ln \left(\frac{2 \ell}{\tau}\right)^{-2}|U(\tau)|^{2} d \tau\right)^{\frac{1}{2}}\left(\int_{0}^{\ell} \tau^{-1} \ln \left(\frac{2 \ell}{\tau}\right)^{-2}\left|U^{\prime}(\tau)\right|^{2} d \tau\right)^{\frac{1}{2}} .
\end{gathered}
$$

The estimates for (2.3) and (2.4) follow similarly.

Lemma 2.3. For all $\beta<0$ there exists $c_{\beta}>0$ such that the following holds. Let $U \in C^{1}([0, \ell])$. Then

$$
U(0)^{2} \leq c_{\beta, \ell} \int_{0}^{\ell} t^{2 \beta+1}\left(U(t)^{2}+U^{\prime}(t)^{2}\right) d t .
$$

Proof. For $\beta<0$ one finds

$$
\begin{aligned}
|U(0)| & \leq|U(t)|+\int_{0}^{t}\left|U^{\prime}(s)\right| d s \\
& \leq|U(t)|+\left(\int_{0}^{t} s^{-1-2 \beta} d s\right)^{1 / 2}\left(\int_{0}^{t} s^{1+2 \beta}\left|U^{\prime}(s)\right|^{2} d s\right)^{1 / 2} \\
& \leq|U(t)|+\frac{1}{\sqrt{2|\beta|}} t^{-\beta}\left(\int_{0}^{\ell} s^{1+2 \beta}\left|U^{\prime}(s)\right|^{2} d s\right)^{1 / 2}
\end{aligned}
$$

Hence it holds that

$$
t^{1+2 \beta}|U(0)|^{2} \leq 2 t^{1+2 \beta}|U(t)|^{2}+\frac{t}{|\beta|} \int_{0}^{\ell} s^{1+2 \beta}\left|U^{\prime}(s)\right|^{2} d s
$$

and for $\beta \in(-1,0)$, when integrated, one finds

$$
\frac{\ell^{2(\beta+1)}}{2(\beta+1)}|U(0)|^{2} \leq 2 \int_{0}^{\ell} t^{1+2 \beta}|U(t)|^{2} d t+\frac{\ell^{2}}{2|\beta|} \int_{0}^{\ell} t^{1+2 \beta}\left|U^{\prime}(t)\right|^{2} d t .
$$

Whenever (2.5) holds for some $\beta_{0}<0$, one can then find a constant $c_{\beta, \ell}>0$ for each $\beta \leq \beta_{0}$. 


\subsection{The variational formulation of the plate problem.}

Definition 2.4. Let $\mathfrak{H}_{\sharp}$ and $\mathfrak{H}_{3}$ be the Hilbert spaces, defined, respectively, as the completions of $C_{c}^{\infty}(\omega)^{2}$ and $C_{c}^{\infty}(\omega)$ with respect to the norms

$$
\left\|v ; \mathfrak{H}_{\sharp}\right\|:=\left(\left\|\rho^{\frac{\alpha}{2}} \mathcal{D}_{\sharp}\left(\nabla_{x}\right) v\right\|_{L^{2}(\omega)}^{2}+\left\|\rho^{\frac{\alpha}{2}} v\right\|_{L^{2}(\omega)}^{2}\right)^{\frac{1}{2}}
$$

and

$$
\left\|w ; \mathfrak{H}_{3}\right\|:=\left(\left\|\rho^{\frac{3 \alpha}{2}} \nabla_{x}^{2} w\right\|_{L^{2}(\omega)}^{2}+\left\|\rho^{\frac{\alpha}{2}} w\right\|_{L^{2}(\omega)}^{2}\right)^{\frac{1}{2}}
$$

Remember that $\rho$ is the horizontal distance to the edge as defined in (1.1).

We consider the integral identity

$$
a_{e}(u, \varphi):=\left(\mathcal{A D}\left(\nabla_{x}\right) u, \mathcal{D}\left(\nabla_{x}\right) \varphi\right)_{\omega}=\mathcal{F}(\varphi) \text { for } \varphi \in \mathfrak{H}=\mathfrak{H}_{\sharp} \times \mathfrak{H}_{3},
$$

where $(., .)_{\omega}$ is the natural scalar product in the Lebesgue space $L^{2}(\omega)$, or its extension up to the duality between proper weighted spaces. On the right-hand side of (2.8) one has $\mathcal{F} \in \mathfrak{H}^{*}$, that is, a continuous linear functional on $\mathfrak{H}$. By $\nabla_{x}^{k} w$, we mean the collection of all $k$ th order derivatives of the function $w$.

Lemma 2.5. Consider the domain $\omega \subset \mathbb{R}^{2}$ and set

$$
r(\rho)=\left(\ln \left(d_{\omega} / \rho\right)\right)^{-1} \text { for } \rho \in\left[0, \frac{1}{2} d_{\omega}\right],
$$

where $d_{\omega}:=2 \max \{\rho(x) ; x \in \omega\}$ which is less than or equal to the diameter of $\omega$.

1. On $C_{c}^{\infty}(\omega)^{2}$ the norm in (2.6) is equivalent to the norm $\|\cdot\|_{\sharp}$ defined by

$$
\begin{gathered}
\|v\|_{\sharp}=\left(\left\|\rho^{\frac{\alpha}{2}} \mathcal{D}_{\sharp}\left(\nabla_{x}\right) v\right\|_{L^{2}(\omega)}^{2}+\left\|\rho^{\frac{\alpha}{2}} R_{\sharp}(\rho) \nabla_{x} v\right\|_{L^{2}(\omega)}^{2}\right. \\
\left.+\left\|\rho^{\frac{\alpha-2}{2}} R_{\sharp}(\rho)^{2} v\right\|_{L^{2}(\omega)}^{2}\right)^{1 / 2},
\end{gathered}
$$

where

$$
R_{\sharp}(\rho)= \begin{cases}r(\rho) & \text { for } \alpha=1, \\ 1 & \text { for } \alpha \neq 1 .\end{cases}
$$

2. On $C_{c}^{\infty}(\omega)$ the norm in (2.7) is equivalent to the norm $\|\cdot\|_{3}$ defined by

$$
\begin{aligned}
\|w\|_{3}=( & \left\|\rho^{\frac{3 \alpha}{2}} \nabla_{x}^{2} w\right\|_{L^{2}(\omega)}^{2}+\left\|\rho^{\frac{3 \alpha-2}{2}} R_{3}^{*}(\rho) \nabla_{x} w\right\|_{L^{2}(\omega)}^{2} \\
& \left.+\left\|\rho^{\frac{3 \alpha-4}{2}} R_{3}(\rho) w\right\|_{L^{2}(\omega)}^{2}+\left\|\rho^{\frac{\alpha}{2}} w\right\|_{L^{2}(\omega)}^{2}\right)^{1 / 2},
\end{aligned}
$$

where

$$
R_{3}^{*}(\rho)=\left\{\begin{array}{ll}
r(\rho) & \text { for } \alpha=\frac{1}{3}, \\
1 & \text { for } \alpha \neq \frac{1}{3},
\end{array} \quad \text { and } R_{3}(\rho)= \begin{cases}r(\rho) & \text { for } \alpha \in\left\{\frac{1}{3}, 1\right\} \\
1 & \text { for } \alpha \notin\left\{\frac{1}{3}, 1\right\} .\end{cases}\right.
$$

Copyright (c) by SIAM. Unauthorized reproduction of this article is prohibited. 
Proof. The estimates

$$
\left\|v ; \mathfrak{H}_{\sharp}\right\| \leq C_{\sharp}\|v\|_{\sharp},\left\|w ; \mathfrak{H}_{3}\right\| \leq C_{3}\|w\|_{3}
$$

one finds directly. Hence we need only verify the inverse relations. These inverse relations use 2-d versions of the Hardy inequalities from Lemma 2.5 that one obtains through the local coordinates $(n, s)$ with $n$ the normal direction and integrating along the tangential direction $s$. The role of $t$ is replaced by $n=\rho(x)$. In several cases we will need a smooth partition of unity $\{\chi, 1-\chi\}$ with $\chi=1$ near the boundary and $\chi=0$ on $\omega \backslash \omega_{\ell}$. We set $S:=\operatorname{support}(1-\chi) \supset \operatorname{support}(|\nabla \chi|)$.

We start with the proof of (2.11) and distinguish five individual cases for $\alpha$.

- $\alpha \in\left(0, \frac{1}{3}\right)$. We find $3 \alpha-2<-1$ and $3 \alpha-4<-1$, and the estimate readily follows from $(2.1)$ :

$$
\left\|\rho^{\frac{3 \alpha-4}{2}} w\right\|_{L^{2}(\omega)} \leq c_{1}\left\|\rho^{\frac{3 \alpha-2}{2}}|\nabla w|\right\|_{L^{2}(\omega)} \leq c_{2}\left\|\rho^{\frac{3 \alpha}{2}}\left|\nabla^{2} w\right|\right\|_{L^{2}(\omega)} .
$$

- $\alpha \in\left(\frac{1}{3}, 1\right)$. We find $3 \alpha-2>-1$ and $3 \alpha-4<-1$. The first estimate follows from (2.1) as in (2.14). For the second one we proceed as follows:

$$
\begin{aligned}
& \left\|\rho^{\frac{3 \alpha-2}{2}}|\nabla w|\right\|_{L^{2}(\omega)} \\
& \quad \leq c_{2}\left(\left\|\rho^{\frac{3 \alpha}{2}}|\nabla(\chi \nabla w)|\right\|_{L^{2}(\omega)}+\||\nabla w|\|_{L^{2}(\omega \cap S)}\right) \\
& \quad \leq c_{3}\left(\left\|\rho^{\frac{3 \alpha}{2}}\left|\nabla^{2} w\right|\right\|_{L^{2}(\omega)}+\|\nabla w \mid\|_{L^{2}(\omega \cap S)}\right) \\
& \quad \leq c_{4}\left(\left\|\rho^{\frac{3 \alpha}{2}}\left|\nabla^{2} w\right|\right\|_{L^{2}(\omega)}+\left\|\left|\nabla^{2} w\right|\right\|_{L^{2}(\omega \cap S)}+\|w\|_{L^{2}(\omega \cap S)}\right) \\
& \quad \leq c_{5}\left(\left\|\rho^{\frac{3 \alpha}{2}}\left|\nabla^{2} w\right|\right\|_{L^{2}(\omega)}+\left\|\rho^{\frac{\alpha}{2}} w\right\|_{L^{2}(\omega)}\right) .
\end{aligned}
$$

- $\alpha \in(1, \infty)$. For both steps we have to split using the partition of unity as in the second step (2.15).

- $\alpha=\frac{1}{3}$. For the first step one uses (2.2) instead of (2.1) to find

$$
\left\|\rho^{\frac{-3}{2}} r(\rho) w\right\|_{L^{2}(\omega)} \leq c_{1}\left\|\rho^{\frac{-1}{2}} r(\rho)|\nabla w|\right\|_{L^{2}(\omega)},
$$

and one finds with (2.3) for the second step that

$$
\left\|\rho^{\frac{-1}{2}} r(\rho)|\nabla w|\right\|_{L^{2}(\omega)} \leq c_{2}\left(\left\|\rho^{\frac{1}{2}}|\nabla(\chi \nabla w)|\right\|_{L^{2}(\omega)}+\||\nabla w|\|_{L^{2}(\omega \cap S)}\right) .
$$

This estimate can be continued as in (2.15).

- $\alpha=1$. Now one uses (2.3) for the first step

$$
\left\|\rho^{\frac{-1}{2}} r(\rho) w\right\|_{L^{2}(\omega)} \leq c_{1}\left(\left\|\rho^{\frac{1}{2}}|\nabla(\chi w)|\right\|_{L^{2}(\omega)}+\|w\|_{L^{2}(\omega \cap S)}\right)
$$

and proves the second step as (2.15).

Copyright $@$ by SIAM. Unauthorized reproduction of this article is prohibited. 
The equivalence of (2.9) and (2.6) is much more subtle. Let us first recall that

$$
\begin{aligned}
\left|\mathcal{D}_{\sharp}(\nabla) v\right|^{2} & =\left(\partial_{1} v_{1}\right)^{2}+\left(\partial_{2} v_{2}\right)^{2}+\frac{1}{2}\left(\partial_{1} v_{2}+\partial_{2} v_{1}\right)^{2} \\
& =\frac{1}{2}|\nabla v|^{2}+\frac{1}{2}\left(\partial_{1} v_{1}\right)^{2}+\frac{1}{2}\left(\partial_{2} v_{2}\right)^{2}+\partial_{1} v_{2} \partial_{2} v_{1}
\end{aligned}
$$

First we consider the case $\alpha=0$. Away from the boundary the thickness is uniform and we may estimate the weight depending on $\rho$ by a positive constant from both sides. An integration by parts will remove the last term in (2.16). Indeed, for $v \in\left(C_{c}^{\infty}\left(\omega_{\ell}\right)\right)^{2}$ we find

$$
\left|\int_{\omega} \partial_{1} v_{2} \partial_{2} v_{1} d x\right|=\left|\int_{\omega} \partial_{1} v_{1} \partial_{2} v_{2} d x\right| \leq \frac{1}{2} \int\left(\left(\partial_{1} v_{1}\right)^{2}+\left(\partial_{2} v_{2}\right)^{2}\right) d x
$$

which implies that

$$
\left\|\mathcal{D}_{\sharp}(\nabla) v\left|\left\|_{L^{2}(\omega)}^{2} \geq \frac{1}{2}\right\|\right| \nabla v \mid\right\|_{L^{2}(\omega)}^{2} .
$$

In order to deal with the weighted case, we introduce the Cartesian components of the strain tensor

$$
\varepsilon_{j k}(v ; x)=\frac{1}{2}\left(\frac{\partial v_{j}}{\partial x_{k}}+\frac{\partial v_{k}}{\partial x_{j}}\right) \quad \text { with } j, k=1,2 .
$$

The components of this tensor in the local coordinates $(n, s)$ are (2.19)

$$
\left\{\begin{array}{l}
\varepsilon_{n n}(n, s)=\partial_{n} v_{n}(n, s) \\
\varepsilon_{s s}(n, s)=J(n, s)^{-1}\left(\partial_{s} v_{s}(n, s)+\kappa(s) v_{n}(n, s)\right) \\
\varepsilon_{n s}(n, s)=\varepsilon_{s n}(n, s)=\frac{1}{2}\left(\partial_{n} v_{s}(n, s)+J(n, s)^{-1}\left(\partial_{s} v_{n}(n, s)-\kappa(s) v_{s}(n, s)\right)\right),
\end{array}\right.
$$

where $\partial_{n}=\frac{\partial}{\partial n}, \partial_{s}=\frac{\partial}{\partial s}$, and $v_{n}$ and $v_{s}$ are the projections of the vector $v$ on the $n$ and $s$-axis, $J(n, s)$ is the Jacobian, and $\kappa(s)$ the arc curvature at the point $s \in \partial \omega$. It is known and can be verified by a direct calculation that

$$
\begin{aligned}
I_{0}: & =\int_{\omega_{\ell}} \rho(x)^{\alpha}\left|\mathcal{D}_{\sharp}\left(\nabla_{x}\right) v(x)\right|^{2} d x=\sum_{j, k=1}^{2} \int_{\omega_{\ell}} \rho(x)^{\alpha} \varepsilon_{j k}(x)^{2} d x \\
& =\int_{\partial \omega} \int_{0}^{\ell} n^{\alpha}\left(\varepsilon_{n n}(n, s)^{2}+2 \varepsilon_{n s}(n, s)^{2}+\varepsilon_{s s}(n, s)^{2}\right) J(n, s) d n d s .
\end{aligned}
$$

An integral close to $\left\|\rho^{\frac{\alpha}{2}}|\nabla v|\right\|_{L^{2}\left(\omega_{\ell}\right)}$ using local coordinates is

$$
\begin{aligned}
I_{1}:= & \int_{\partial \omega} \int_{0}^{\ell} n^{\alpha}\left(\left(\partial_{n} v_{n}\right)^{2}+\frac{1}{2}\left(\partial_{n} v_{s}+J^{-1} \partial_{s} v_{n}\right)^{2}+J^{-2}\left(\partial_{s} v_{s}\right)^{2}\right) J d n d s \\
= & \int_{\partial \omega} \int_{0}^{\ell} n^{\alpha}\left(\varepsilon_{n n}^{2}+2\left(\varepsilon_{n s}+\frac{1}{2} \kappa J^{-1} v_{s}\right)^{2}+\left(\varepsilon_{s s}-\kappa J^{-1} v_{n}\right)^{2}\right) J d n d s \\
= & I_{0}+\int_{\partial \omega} \int_{0}^{\ell} n^{\alpha}\left(2 \kappa\left(\varepsilon_{n s} v_{s}-\varepsilon_{s s} v_{n}\right)+\kappa^{2} J^{-1}\left(\frac{1}{2} v_{s}^{2}+v_{n}^{2}\right)\right) d n d s \\
& \leq I_{0}+c_{1} \sqrt{I_{0}}\left\|\rho^{\frac{\alpha}{2}} v\right\|_{L^{2}\left(\omega_{\ell}\right)}+c_{2}\left\|\rho^{\frac{\alpha}{2}} v\right\|_{L^{2}\left(\omega_{\ell}\right)}^{2} \leq c_{3}\left\|v ; \mathfrak{H}_{\sharp}\right\|^{2} .
\end{aligned}
$$

Copyright $@$ by SIAM. Unauthorized reproduction of this article is prohibited. 
Indeed one finds that

$$
\begin{aligned}
I_{1}= & \int_{\partial \omega} \int_{0}^{\ell} n^{\alpha} \frac{1}{2}\left|\nabla_{n, s}\left(v_{n}, v_{s}\right)\right|^{2} J d n d s \\
& +\int_{\partial \omega} \int_{0}^{\ell} n^{\alpha}\left(J^{-2}-1\right)\left(\left(\partial_{s} v_{n}\right)^{2}+\left(\partial_{s} v_{s}\right)^{2}\right) J d n d s \\
& +\int_{\partial \omega} \int_{0}^{\ell} n^{\alpha}\left(\frac{1}{2} J\left(\partial_{n} v_{n}\right)^{2}+\frac{1}{2} J^{-1}\left(\partial_{s} v_{s}\right)^{2}\right) d n d s \\
& +\int_{\partial \omega} \int_{0}^{\ell} n^{\alpha}\left(-\partial_{s} v_{n} \partial_{n} v_{s}\right) d n d s .
\end{aligned}
$$

We name these four integrals in (2.22)-(2.25), respectively, $I_{11}, I_{12}, I_{13}$, and $I_{14}$. By taking $\ell$ small enough since $J$ goes to 1 when $\ell$ goes to 0 , one finds that one may take $\delta>0$ as small as one needs and get

$$
\left|I_{12}\right| \leq \delta I_{11} .
$$

The choice $\delta=\frac{1}{2}$ will suffice. For the third item (2.24) one finds by (2.21) that

$$
I_{13} \leq \frac{1}{2} I_{1} .
$$

For the last item (2.25) an integration by parts gives

$$
\begin{aligned}
\left|I_{14}\right|= & \left|\int_{\partial \omega} \int_{0}^{\ell} n^{\alpha} \partial_{n} v_{s} \partial_{s} v_{n} d n d s\right| \\
= & \left|-\int_{\partial \omega} \int_{0}^{\ell}\left(\partial_{n}\left(n^{\alpha}\right) v_{n} \partial_{s} v_{s}+n^{\alpha} \partial_{s} v_{s} \partial_{n} v_{n}\right) d n d s\right| \\
\leq & c_{4}\left|\int_{\partial \omega} \int_{0}^{\ell} n^{\alpha-1} v_{n} \partial_{s} v_{s} d n d s\right| \\
& +\int_{\partial \omega} \int_{0}^{\ell} n^{\alpha}\left(\frac{1}{2} J\left(\partial_{n} v_{n}\right)^{2}+\frac{1}{2} J^{-1}\left(\partial_{s} v_{s}\right)^{2}\right) d n d s .
\end{aligned}
$$

Hence we have found

$$
\begin{aligned}
\left|I_{1}-I_{11}\right| & \leq \delta I_{11}+2 I_{13}+c_{4}\left|\int_{\partial \omega} \int_{0}^{\ell} n^{\alpha-1} v_{n} \partial_{s} v_{s} d n d s\right| \\
& \leq \delta I_{11}+I_{1}+c_{4}\left|\int_{\partial \omega} \int_{0}^{\ell} n^{\alpha-1} v_{n} \partial_{s} v_{s} d n d s\right| .
\end{aligned}
$$

For $\alpha \neq 1$ we may use (2.1) to derive

$$
\begin{aligned}
& \left|\int_{\partial \omega} \int_{0}^{\ell} n^{\alpha-1} v_{n} \partial_{s} v_{s} d n d s\right| \leq\left\|\rho^{\frac{\alpha-2}{2}} v_{n}\right\|_{L^{2}\left(\omega_{\ell}\right)}\left\|\rho^{\frac{\alpha}{2}} \partial_{s} v_{s}\right\|_{L^{2}\left(\omega_{\ell}\right)} \\
& \leq c_{5}\left(\left\|\rho^{\frac{\alpha}{2}} v_{n}\right\|_{L^{2}\left(\omega_{\ell}\right)}+\left\|\rho^{\frac{\alpha}{2}} \partial_{n} v_{n}\right\|_{L^{2}\left(\omega_{\ell}\right)}\right)\left\|\rho^{\frac{\alpha}{2}} \partial_{s} v_{s}\right\|_{L^{2}\left(\omega_{\ell}\right)} \leq c_{5}\left\|v ; \mathfrak{H}_{\sharp}\right\|^{2} .
\end{aligned}
$$

So with (2.21) again one concludes from (2.27) that

$$
\left\|\rho^{\frac{\alpha}{2}}|\nabla v|\right\|_{L^{2}\left(\omega_{\ell}\right)}^{2} \leq c I_{11} \leq \frac{c}{1-\delta}\left(2 I_{1}+c_{4} c_{5}\left\|v ; \mathfrak{H}_{\sharp}\right\|^{2}\right) \leq \frac{c}{1-\delta}\left(2 c_{3}+c_{4} c_{5}\right)\left\|v ; \mathfrak{H}_{\sharp}\right\|^{2} .
$$


Together with (2.17) it follows for $\alpha \in(0, \infty) \backslash\{1\}$ that

$$
\left\|\rho^{\frac{\alpha}{2}}|\nabla v|\right\|_{L^{2}(\omega)}^{2} \leq C\left\|v ; \mathfrak{H}_{\sharp}\right\|^{2} .
$$

For $\alpha=1$ one may use (2.3) and replace (2.28) by

$$
\begin{aligned}
& \left|\int_{\partial \omega} \int_{0}^{\ell} r(n) v_{n} \partial_{s} v_{s} d n d s\right| \leq\left\|\rho^{\frac{-1}{2}} r(\rho) v_{n}\right\|_{L^{2}\left(\omega_{\ell}\right)}\left\|\rho^{\frac{1}{2}} \partial_{s} v_{s}\right\|_{L^{2}\left(\omega_{\ell}\right)} \\
& \leq c_{5}\left(\left\|\rho^{\frac{1}{2}} v_{n}\right\|_{L^{2}\left(\omega_{\ell}\right)}+\left\|\rho^{\frac{\alpha}{2}} \partial_{n} v_{n}\right\|_{L^{2}\left(\omega_{\ell}\right)}\right)\left\|\rho^{\frac{\alpha}{2}} \partial_{s} v_{s}\right\|_{L^{2}\left(\omega_{\ell}\right)} \leq c_{5}\left\|v ; \mathfrak{H}_{\sharp}\right\|^{2} .
\end{aligned}
$$

This would allow us to estimate $\left\|\rho^{\frac{1}{2}} r(\rho)|\nabla v|\right\|_{L^{2}(\omega)}^{2}$. In order to do so one has to check that in all the estimates (2.20) until (2.27) one may add an extra weight $r(\rho)$, respectively $r(n)$, without having crucial differences. Indeed, this holds true and the only nontrivial change appears in (2.26) by the derivation of $n^{\alpha} r(n)$ instead of $n^{\alpha}$. However, since

$$
\left|\partial_{n}\left(n^{\alpha} r(n)\right)\right|=\left|n^{\alpha-1}\left(\alpha^{2} r(n)+r(n)^{2}\right)\right| \leq c n^{\alpha-1} r(n)
$$

also, that step still holds true. Another change is necessary for the zero order norm of $v$. Since we need to estimate the zero order norm of $v$ by $\left\|\rho^{\frac{1}{2}} r(\rho)|\nabla v|\right\|_{L^{2}(\omega)}$, we have to use (2.4), meaning

$$
\left\|\rho^{\frac{-1}{2}} r(\rho)^{2} v\right\|_{L^{2}\left(\omega_{\ell}\right)} \leq c\left\|\rho^{\frac{1}{2}} r(\rho) \mid \nabla v\right\|_{L^{2}\left(\omega_{\ell}\right)}
$$

for $v \in C_{c}^{\infty}\left(\omega_{\ell}\right)^{2}$, and hence this weaker zero order norm is appropriate in (2.9) for $\alpha=1$.

Remark 2.6. The logarithmical factors (2.10)-(2.12) appear in (2.11) due to the application of the Hardy inequalities (2.2) and (2.3), which are known to be optimal. The factor (2.10) in front of the gradient $\nabla_{x} v$ in (2.9) is caused only by our way of verification. As seen in the last lines of the proof, it also results in the factor $R_{\sharp}^{2}(n)$ in front of $v$ in (2.9). The authors do not know if these weights are optimal. The same optimality question arises in many variants of weighted Korn inequalities on 2-d domains and remains to be open (cf. [11, 31] and others).

With a similar argument as above, we derive from a Hardy-type inequality, namely (2.5), the following sufficient condition for the existence of a trace in $L^{2}(\partial \omega)$.

Lemma 2.7. For $\beta<0$ there exists $C_{\beta}>0$ such that every $u \in C^{\infty}(\bar{\omega})$ satisfies the estimate

$$
\int_{\partial \omega} u(x)^{2} d s_{x} \leq C_{\beta} \int_{\omega} \rho(x)^{2 \beta+1}\left(\left|\nabla_{x} u(x)\right|^{2}+u(x)^{2}\right) d x
$$

possibly including $\infty$ on the right in the case $\beta \leq-1$.

If $u \in C^{\infty}(\omega)$ and the right-hand side of $(2.30)$ is bounded for some $\beta<0$, then the trace of $u$ on $\partial \omega$ exists in $L^{2}(\partial \omega)$.

Proof. The estimate follows directly by Lemma 2.3 .

Lemmas 2.5 and 2.7 guarantee that first, for $\alpha<1$, a vector function $v \in \mathfrak{H}_{\sharp}$ keeps the boundary condition

$$
v(x)=0 \text { for } x \in \partial \omega .
$$

Copyright (C) by SIAM. Unauthorized reproduction of this article is prohibited. 
Second, in the case that $\alpha \leq \frac{1}{3}$ a function $w \in \mathfrak{H}_{3}$ satisfies conditions (1.8). Similarly, in the case $\alpha<1$ a function $w \in \mathfrak{H}_{3}$ satisfies condition (1.9).

Lemma 2.8. Let $\mathfrak{H}_{\sharp}$ and $\mathfrak{H}_{3}$ be as in Definition 2.4.

1. A vector-function $v \in C^{\infty}(\bar{\omega})^{2}$ belongs to $\mathfrak{H}_{\sharp}$ if $\alpha \geq 1$.

2. A function $w \in C^{\infty}(\bar{\omega})$ belongs to $\mathfrak{H}_{3}$ if $\alpha \geq 1$.

3. A function $w \in C^{\infty}(\bar{\omega}) \cap C_{0}(\bar{\omega})$ belongs to $\mathfrak{H}_{3}$ if $\alpha \geq \frac{1}{3}$.

Proof. Let $\chi_{\varepsilon} \in C_{c}^{\infty}(\omega)$ be a cut-off function such that $\chi_{\varepsilon}(x)=1$ for $x \in \omega \backslash \omega_{\varepsilon}$, $\chi_{\varepsilon}(x)=0$ for $x \in \omega_{\frac{\varepsilon}{2}}$, and $0 \leq \chi_{\varepsilon} \leq 1$, where $\varepsilon>0$ is a small parameter. One may set $\chi_{\varepsilon}(x)=\chi(\rho(x) / \varepsilon)$ inside $\omega_{d}$, where $\chi$ is a standard cut-off function,

$$
\chi \in C^{\infty}(\mathbb{R}), \quad 0 \leq \chi \leq 1 \text { with } \begin{cases}\chi(t)=0 & \text { for } t \leq \frac{1}{2} \\ \chi(t)=1 & \text { for } t \geq 1\end{cases}
$$

and extend $\chi_{\varepsilon}$ by one to $\omega \backslash \omega_{d}$. Clearly,

$$
\left|\nabla_{x}^{k} \chi_{\varepsilon}(x)\right| \leq C_{k} \varepsilon^{-k}, \quad x \in \omega, \quad k=1,2, \ldots .
$$

We readily observe the inequalities

$$
\begin{gathered}
\left\|\left(1-\chi_{\varepsilon}\right) v ; \mathfrak{H}_{\sharp}\right\|^{2} \\
\leq C \int_{\omega} \rho(x)^{\alpha}\left(\left|\nabla_{x} \chi_{\varepsilon}(x)\right|^{2}|v(x)|^{2}+\left(1-\chi_{\varepsilon}(x)\right)^{2}\left(\left|\nabla_{x} v(x)\right|^{2}+|v(x)|^{2}\right)\right) d x \\
\leq C_{v} \int_{0}^{\varepsilon} n^{\alpha}\left(\varepsilon^{-2}+1\right) d n \leq C_{v}^{\prime} \varepsilon^{\alpha-1} ; \\
\left\|\left(1-\chi_{\varepsilon}\right) w ; \mathfrak{H}_{3}\right\|^{2} \\
\leq C \int_{\omega}\left(\rho(x)^{3 \alpha}\left(\left|\nabla_{x}^{2} \chi_{\varepsilon}(x)\right|^{2}|w(x)|^{2}+\left|\nabla_{x} \chi_{\varepsilon}(x)\right|^{2}\left|\nabla_{x} w(x)\right|^{2}\right)\right. \\
\left.+\left(1-\chi_{\varepsilon}(x)\right)^{2}\left(\rho(x)^{3 \alpha}\left|\nabla_{x}^{2} w(x)\right|^{2}+\rho(x)^{\alpha}|w(x)|^{2}\right)\right) d x \\
\leq C_{w} \int_{0}^{\varepsilon} n^{3 \alpha}\left(\varepsilon^{-4}+\varepsilon^{-2}+1\right) d n \leq C_{w}^{\prime} \varepsilon^{3 \alpha-3} .
\end{gathered}
$$

Furthermore, under the condition $|w(x)| \leq C_{w} \rho(x)$, the latter bound reduces to $C_{0} \varepsilon^{3 \alpha-1}$, since then the term $\varepsilon^{-4}$ in the last integral is replaced by $\varepsilon^{-2}$. Thus, the vector function $v$ and the function $w$ can be approximated, respectively, by $\chi_{\varepsilon} v \in$ $C_{c}^{\infty}(\omega)^{2}$ in the norm (2.6) and by $\chi_{\varepsilon} w \in C_{c}^{\infty}(\omega)$ in the norm (2.7), provided the bounds in (2.33)-(2.34) are infinitesimal as $\varepsilon \rightarrow 0$. This completes the proof of the three claims of the lemma, except, respectively, for the cases $\alpha=1, \alpha=1$, and $\alpha=\frac{1}{3}$.

To complete the proof, we consider the new cut-off function $\chi_{\varepsilon}^{0}$ defined by

$$
\chi_{\varepsilon}^{0}(x)=\chi(\ln (\rho(x)) /|\ln \varepsilon|)
$$

inside $\omega_{d}$. Notice that $\chi_{\varepsilon}^{0}(x)$ goes from 0 to 1 for $\rho$ from $\sqrt{\varepsilon}$ to $\varepsilon$, that is, the support of $\left|\nabla_{x} \chi_{\varepsilon}^{0}\right|$ by (2.32), belongs to $\bar{\omega}_{\sqrt{\varepsilon}} \backslash \omega_{\varepsilon}$ and the following estimates are valid:

$$
\left|\nabla_{x} \chi_{\varepsilon}^{0}(x)\right| \leq C_{1}|\ln \varepsilon|^{-1} \rho(x)^{-1} \text { and }\left|\nabla_{x}^{2} \chi_{\varepsilon}^{0}(x)\right| \leq C_{2}|\ln \varepsilon|^{-1} \rho(x)^{-2} .
$$

Copyright (c) by SIAM. Unauthorized reproduction of this article is prohibited. 
We now have

$$
\left\|\left(1-\chi_{\varepsilon}^{0}\right) v ; \mathfrak{H}_{\sharp}\right\|^{2} \leq C_{v}\left(\int_{\varepsilon}^{\sqrt{\varepsilon}} n|\ln \varepsilon|^{-2} n^{-2} d n+\int_{0}^{\sqrt{\varepsilon}} n d n\right) \leq \frac{C}{|\ln \varepsilon|}
$$

that provides the convergence of $\chi_{\varepsilon}^{0} v \in C_{c}^{\infty}(\omega)$ to $v \in C_{c}^{\infty}(\bar{\omega})$ in the norm of $\mathcal{H}_{\sharp}$, that is, the assertion 1 is verified. The assertions 2 and 3 follow from the calculation

$$
\left\|\left(1-\chi_{\varepsilon}^{0}\right) w ; \mathfrak{H}_{3}\right\|^{2} \leq C_{w}\left(\int_{\varepsilon}^{\sqrt{\varepsilon}} n^{3}|\ln \varepsilon|^{-2}\left(n^{-4}+n^{-2}\right) d n+\int_{0}^{\sqrt{\varepsilon}} n^{3} d n\right) \leq \frac{C}{|\ln \varepsilon|}
$$

and under the condition $|w(x)| \leq C \rho(x)$, from the calculation

$$
\left\|\left(1-\chi_{\varepsilon}^{0}\right) w ; \mathfrak{H}_{3}\right\|^{2} \leq C_{w}\left(\int_{\varepsilon}^{\sqrt{\varepsilon}} n|\ln \varepsilon|^{-2} n^{-2} d n+\int_{0}^{\sqrt{\varepsilon}} n d n\right) \leq \frac{C}{|\ln \varepsilon|} .
$$

The main result of this section follows from the lemmas proven above.

TheOREM 2.9. Let $\mathfrak{H}_{\sharp}$ and $\mathfrak{H}_{3}$ be as in Definition 2.4.

1. A vector function $v \in \mathfrak{H}_{\sharp}$ satisfies the boundary condition (2.31) if and only if $\alpha<1$.

2. A function $w \in \mathfrak{H}_{3}$ satisfies the boundary condition (1.8) (resp., (1.9)) if and only if $\alpha<\frac{1}{3}$ (resp., $\alpha<1$ ).

2.3. Solvability of the singular thin plate problem. First of all, we consider the case $\mathcal{A}_{3 \sharp}(x)=\mathcal{A}_{\sharp 3}(x)^{\top}=\mathbf{0}_{3}$ (see (1.27)), i.e., problem (2.8) splits into the 2-d elasticity problem

$$
a_{e \sharp}\left(v, \varphi_{\sharp}\right):=\left(\mathcal{A}_{\sharp \sharp} \mathcal{D}_{\sharp}\left(\nabla_{x}\right) v, \mathcal{D}_{\sharp}\left(\nabla_{x}\right) \varphi_{\sharp}\right)_{\omega}=\mathcal{F}_{\sharp}\left(\varphi_{\sharp}\right) \text { for } \varphi_{\sharp} \in \mathfrak{H}_{\sharp}
$$

for the longitudinal displacement vector $v=\left(v_{1}, v_{2}\right)^{\top}$ and the following problem on the deflection $w$ :

$$
a_{e 3}\left(w, \varphi_{3}\right):=\left(\mathcal{A}_{33} \mathcal{D}_{3}\left(\nabla_{x}\right) w, \mathcal{D}_{3}\left(\nabla_{x}\right) \varphi_{3}\right)_{\omega}=\mathcal{F}_{3}\left(\varphi_{3}\right) \text { for } \varphi_{3} \in \mathfrak{H}_{3} .
$$

Since evidently the $3 \times 3$ matrices $\mathcal{A}_{\sharp \sharp}$ and $\mathcal{A}_{33}$ are symmetric and positive definite due to the representation formula (1.19) and the same properties of $A(y, \zeta)$, we see that, in view of the structure of the differential operator (1.18) and the formulas (1.25)-(1.26), the quadratic forms $a_{e \sharp}(v, v)$ and $a_{e 3}(w, w)$ may degenerate only on the linear space

$$
\begin{aligned}
& \mathcal{R}_{\sharp}=\left\{v=\left(v_{1}, v_{2}\right)^{\top}: v_{1}(x)=c_{1}-c_{0} x_{2}, \quad v_{2}(x)=c_{2}+c_{0} x_{1}\right\}, \\
& \mathcal{R}_{3}=\left\{w: w(x)=c_{0}+c_{1} x_{1}+c_{2} x_{2}\right\},
\end{aligned}
$$

where $c_{0}, c_{1}$ and $c_{3}$ are constant. There are no nontrivial elements $r_{\sharp} \in \mathcal{R}_{\sharp}$ and $r_{3} \in \mathcal{R}_{3}$ which, respectively, satisfy the boundary conditions (2.31) and (1.9). Hence, by Theorem 2.9, the quadratic forms $a_{e \sharp}$ and $a_{e 3}$ are positive definite in the case $\alpha<1$ but they are only positive in the case $\alpha \geq 1$ with, respectively, the null-spaces (2.37) and (2.38). Notice that for $\alpha \geq 1$, problems (2.35) and (2.36) have only a solution under the compatibility conditions

$$
\mathcal{F}_{\sharp}\left(r_{\sharp}\right)=0 \text { for all } r_{\sharp} \in \mathcal{R}_{\sharp}, \quad \mathcal{F}_{3}\left(r_{3}\right)=0 \text { for all } r_{3} \in \mathcal{R}_{3} .
$$

Now we prove the similar result for the coupled system. 
TheOREm 2.10. Consider the quadratic form $a_{e}(u, u)$, with $a_{e}$ defined in (2.8).

1. If $\alpha<1$, then $a_{e}(u, u)$ is positive definite ${ }^{1}$ and the problem (2.8) has a unique solution $u \in \mathfrak{H}=\mathfrak{H}_{\sharp} \times \mathfrak{H}_{3}$ for any $\mathcal{F} \in \mathfrak{H}^{*}$. Moreover, there exist $c>0$ such that for any $\mathcal{F} \in \mathfrak{H}^{*}$ and corresponding solution u the estimate

$$
\|u ; \mathfrak{H}\| \leq c\left\|\mathcal{F} ; \mathfrak{H}^{*}\right\|
$$

is valid.

2. If $\alpha \geq 1$, the quadratic form $a_{e}(u, u)$ is only positive and problem (2.8) has a solution $u \in \mathfrak{H}$ if and only if the functional $\mathcal{F}=\left(\mathcal{F}_{\sharp}, \mathcal{F}_{3}\right) \in \mathfrak{H}^{*}$ satisfies the compatibility conditions (2.39). The solution $u$ is defined up to an addition $r=\left(r_{\sharp}, r_{3}\right) \in \mathcal{R}=\left(\mathcal{R}_{\sharp}, \mathcal{R}_{3}\right)$ and under the orthogonality conditions

$$
\left(\rho^{\alpha} u, r\right)_{\omega}=0 \text { for all } r \in \mathcal{R}
$$

it becomes unique and admits the estimate (2.40).

Proof. We shall verify the inequality

$$
\left\|\rho^{\frac{\alpha}{2}} \mathcal{D}_{\sharp}\left(\nabla_{x}\right) v\right\|_{L^{2}(\omega)}^{2}+\left\|\rho^{\frac{3 \alpha}{2}} \nabla_{x}^{2} w\right\|_{L^{2}(\omega)}^{2} \leq c a_{e}(u, u) \text { for } u \in \mathfrak{H},
$$

which, by the Lax-Milgram lemma or the Riesz representation theorem, together with Theorem 2.9 and the above consideration, provides the formulated assertions.

The inequality (2.42) is true if we prove that the matrix (1.19) is positive definite and satisfies a properly weighted inequality. Let $\xi=\left(\xi_{\sharp}^{\top}, \xi_{3}^{\top}\right)^{\top} \in \mathbb{R}^{6}$ and set

$$
\Xi(x)=\left(h(x)^{-\frac{1}{2}} \xi_{\sharp}^{\top}, h(x)^{-\frac{3}{2}} \xi_{3}^{\top}\right)^{\top} .
$$

By (1.19), we have for some $C_{A}>0$ that

$$
\begin{gathered}
\Xi(x)^{\top} \mathcal{A}(x) \Xi(x) \\
=\int_{-h_{-}(x)}^{h_{+}(x)} h(x)^{-1}\left(\xi_{\sharp}-\sqrt{2} h(x)^{-1} \zeta \xi_{3}\right)^{\top} A(x, \zeta)\left(\xi_{\sharp}-\sqrt{2} h(x)^{-1} \zeta \xi_{3}\right) d \zeta \\
=\int_{\Upsilon(x)}\left(\xi_{\sharp}-\sqrt{2} z \xi_{3}\right)^{\top} A(x, h(x) z)\left(\xi_{\sharp}-\sqrt{2} z \xi_{3}\right) d z \\
\geq C_{A}\left\|\xi_{\sharp}-\sqrt{2} z \xi_{3}\right\|_{L^{2}(\Upsilon(x))}^{2},
\end{gathered}
$$

where

$$
\Upsilon(x)=\left[-h_{-}(x) / h(x), h_{+}(x) / h(x)\right] .
$$

Due to (1.22) and (1.2), the length of the interval $\Upsilon(x)$ is 1 . Setting $a(x)=$ $\frac{h_{+}(x)-h_{-}(x)}{2 h(x)}$ we find

$$
\left\|\xi_{\sharp}-\sqrt{2} z \xi_{3}\right\|_{L^{2}(\Upsilon(x))}^{2}=\left|\xi_{\sharp}-\sqrt{2} a(x) \xi_{3}\right|^{2}+\frac{1}{6}\left|\xi_{3}\right|^{2} .
$$

\footnotetext{
${ }^{1}$ The miscalculation mentioned in Remark 1.1 leads to the wrong restriction $\alpha<\frac{4}{3}$ in [19].
}

Copyright (C) by SIAM. Unauthorized reproduction of this article is prohibited. 
Since $|a(x)| \leq c_{h}$ with $c_{h}$ as in (1.23) we derive from (2.43) that there is $C_{\mathcal{A}}>0$ such that

$$
\Xi(x)^{\top} \mathcal{A}(x) \Xi(x) \geq C_{\mathcal{A}}|\xi|^{2} \quad \text { for all } \xi \in \mathbb{R}^{6},
$$

or, equivalently, that

$$
\xi^{\top} \mathcal{A}(x) \xi \geq C_{\mathcal{A}}\left(h(x)\left|\xi_{\sharp}\right|^{2}+h(x)^{3}\left|\xi_{3}\right|^{2}\right) \quad \text { for all } \xi \in \mathbb{R}^{6},
$$

which proves (2.42).

\section{The spectra of these problems.}

3.1. Longitudinal oscillations of the plate. The imbedding $\mathfrak{H}_{\sharp} \subset \mathfrak{L}_{\sharp}$ is compact where $\mathfrak{L}_{\sharp}=\mathfrak{L} \times \mathfrak{L}$ and $\mathfrak{L}$ is a weighted Lebesgue space with the norm defined by

$$
\|w ; \mathfrak{L}\|=\left\|\rho^{\frac{\alpha}{2}} w\right\|_{L^{2}(\omega)} .
$$

Indeed, if the support of $v \in \mathfrak{H}_{\sharp}$ belongs to $\omega \backslash \omega_{\varepsilon}$, then $v \in H^{1}\left(\omega \backslash \omega_{\varepsilon}\right)$ since $\rho(x) \geq$ $\varepsilon>0$ for $x \in \omega \backslash \omega_{\varepsilon}$ and $H^{1}\left(\omega \backslash \omega_{\varepsilon}\right)$ embeds compactly into $L^{2}\left(\omega \backslash \omega_{\varepsilon}\right)$.

Furthermore, if support $(v) \subset \bar{\omega}_{\varepsilon}$, then by Lemma 2.5.1,

$$
\left\|v ; \mathfrak{L}_{\sharp}\right\| \leq C \varepsilon\left(1+\delta_{\alpha, 1}|\ln \varepsilon|\right)\left\|\rho^{\frac{\alpha-2}{2}} R_{\sharp}(\rho) v\right\|_{L^{2}\left(\omega_{\varepsilon}\right)} \leq c \varepsilon\left(1+\delta_{\alpha, 1}|\ln \varepsilon|\right)\left\|v ; \mathfrak{H}_{\sharp}\right\|,
$$

where $\delta_{\alpha, 1}=1$ if $\alpha=1$ and $\delta_{\alpha, 1}=0$, otherwise. In other words, this embedding operator $(\varepsilon \rightarrow+0$ in (3.2)) can be approximated in the operator norm by compact operators and, therefore, it is compact as well.

The weak formulation of the spectral problem (1.31) reads as

$$
\left(\mathcal{A}_{\sharp \sharp} \mathcal{D}_{\sharp}\left(\nabla_{x}\right) v, \mathcal{D}_{\sharp}\left(\nabla_{x}\right) \varphi_{\sharp}\right)_{\omega}=\mu\left(\gamma v, \varphi_{\sharp}\right)_{\omega} \text { for all } \varphi_{\sharp} \in \mathfrak{H}_{\sharp} .
$$

Equipping the Hilbert space $\mathfrak{H}_{\sharp}$ with the specific scalar product

$$
\left\langle v, \varphi_{\sharp}\right\rangle_{\sharp}:=\left(\mathcal{A}_{\sharp \sharp} \mathcal{D}_{\sharp}\left(\nabla_{x}\right) v, \mathcal{D}_{\sharp}\left(\nabla_{x}\right) \varphi_{\sharp}\right)_{\omega}+\left(\gamma v, \varphi_{\sharp}\right)_{\omega}
$$

(cf. Lemma 2.5, Theorem 2.10 and formulas (1.2), (1.25)), we reduce to the abstract equation

$$
T_{\sharp} v=\tau v \quad \text { in } \mathfrak{H}_{\sharp},
$$

where $\tau$ is the new spectral parameter

$$
\tau=(1+\mu)^{-1}
$$

and $T_{\sharp}$ is a positive and continuous, symmetric, therefore, self-adjoint operator

$$
\left\langle T_{\sharp} v, \varphi_{\sharp}\right\rangle_{\sharp}=\left(\gamma v, \varphi_{\sharp}\right)_{\omega}, \quad v, \varphi_{\sharp} \in \mathfrak{H}_{\sharp} .
$$

The operator $T_{\sharp}$ inherits the compactness from the embedding $\mathfrak{H}_{\sharp} \subset \mathfrak{L}_{\sharp}$. Thus, [2, Thm. 10.1.5] ensures that the spectrum $\sigma\left(T_{\sharp}\right)$ consists of the essential spectrum $\sigma_{\text {ess }}\left(T_{\sharp}\right)=\{0\}$ (the only point) and the discrete spectrum $\sigma_{\text {dis }}\left(T_{\sharp}\right)$ which forms a sequence of positive eigenvalues that converge to 0 . The formula (3.6) establishes

Copyright $@$ by SIAM. Unauthorized reproduction of this article is prohibited. 
a relationship between the spectra of problem (3.3) and equation (3.5), while the point $\mu=0$ turns into the infinitely remote point $\lambda_{\infty}$, which does not influence the spectrum. We are in position to formulate the main result of this section.

TheOREM 3.1. For any $\alpha$, the spectrum of problem (3.3) is discrete and forms the eigenvalue sequence

$$
\mu_{1} \leq \mu_{2} \leq \mu_{3} \leq \cdots \leq \mu_{p} \leq \cdots \longrightarrow+\infty
$$

(counted including multiplicity). Moreover,

- if $\alpha<1$, all eigenvalues in (3.8) are positive;

- if $\alpha \geq 1$, then we have $\mu_{4}>0$ and $\mu_{1}=\mu_{2}=\mu_{3}=0$ with the eigenspace (2.37).

3.2. Transversal oscillations of the plate. First, we assume that $\mathcal{A}_{3 \sharp}(x)=$ $\mathcal{A}_{\sharp 3}(x)^{\top}=\mathbf{0}_{3}$ so that the third equation

$$
\mathcal{D}_{3}\left(-\nabla_{x}\right)^{\top} \mathcal{A}_{33}(x) \mathcal{D}_{3}\left(\nabla_{x}\right) w(x)=\lambda \gamma(x) w(x) \text { for } x \in \omega
$$

splits from the spectral problem (1.29) and admits the variational formulation

$$
\left(\mathcal{A}_{33} \mathcal{D}_{3}\left(\nabla_{x}\right) w, \mathcal{D}_{3}\left(\nabla_{x}\right) \varphi_{3}\right)_{\omega}=\lambda\left(\gamma w, \varphi_{3}\right)_{\omega} \text { for } \varphi_{3} \in \mathfrak{H}_{3} .
$$

Note that in the case of the geometrical symmetry (1.27) and the isotropic material (1.28), the problem (3.9) takes the form of Mikhlin's spectral problem in section 1.1.

With the same argument as above, Lemma 2.5.2 ensures that the imbedding $\mathfrak{H}_{3} \subset \mathfrak{L}_{3}=\mathfrak{L}$ is compact under the condition $\alpha<2$. To see this, one needs to compare the weights $\rho^{\frac{\alpha}{2}}$ in the norm (3.1) and $\rho^{\frac{3 \alpha-4}{2}} R_{3}(\rho)$ in the last norm in (2.11). Indeed, the restriction $\alpha<2$ guarantees that the second exponent of $\rho$ is smaller than the first one, which in turn provides the compactness (cf. section 3.1). As a result, we conclude that the spectrum of problem (3.10) is discrete. We may recall the result of Mikhlin mentioned in section 3.1.

We now formulate the full assertion on the spectral problem (3.10) but complete the proof in the next section for the coupled problem (1.17).

THEOREM 3.2. The spectrum of the problem (3.10) is discrete if and only if $\alpha<2$. The corresponding eigenvalue sequence

$$
\lambda_{1} \leq \lambda_{2} \leq \lambda_{3} \leq \cdots \leq \lambda_{p} \leq \cdots \longrightarrow+\infty
$$

is strictly positive in the case $\alpha<1$. For $\alpha \in[1,2)$, we have $\lambda_{4}>0$ and $\lambda_{1}=\lambda_{2}=$ $\lambda_{3}=0$ with the eigenspace (2.38). If $\alpha \geq 2$, the essential spectrum of the problem (3.10) is not empty.

3.3. The reduction of the system to an integro-differential equation. A weak solution to problem (1.29) is $\lambda \in \mathbb{C}$ and $u=\left(v_{1}, v_{2}, w\right)^{\top} \in \mathfrak{h} \backslash\{0\}$ such that

$$
\left(\mathcal{A D}\left(\nabla_{x}\right) u, \mathcal{D}\left(\nabla_{x}\right) \varphi\right)_{\omega}=\lambda(\gamma w, \varphi)_{\omega} \text { for all } \varphi \in \mathfrak{H}
$$

The right-hand side of the integral identity (3.12) does not contain the components $v_{1}$ and $v_{2}$, which makes it impossible to directly apply the theory of selfadjoint operators in Hilbert space. In order to improve the situation, we write the 
problem (3.12) componentwise

$$
\begin{gathered}
\left(\mathcal{A}_{\sharp \sharp} \mathcal{D}_{\sharp}\left(\nabla_{x}\right) v, \mathcal{D}_{\sharp}\left(\nabla_{x}\right) \varphi_{\sharp}\right)_{\omega}=-\left(\mathcal{A}_{\sharp 3} \mathcal{D}_{3}\left(\nabla_{x}\right) w, \mathcal{D}_{\sharp}\left(\nabla_{x}\right) \varphi_{\sharp}\right)_{\omega} \text { for all } \varphi_{\sharp} \in \mathfrak{H}_{\sharp}, \\
\left(\mathcal{A}_{3 \sharp} \mathcal{D}_{\sharp}\left(\nabla_{x}\right) v, \mathcal{D}_{3}\left(\nabla_{x}\right) \varphi_{3}\right)_{\omega}+\left(\mathcal{A}_{33} \mathcal{D}_{3}\left(\nabla_{x}\right) w, \mathcal{D}_{3}\left(\nabla_{x}\right) \varphi_{3}\right)_{\omega} \\
=\lambda\left(\gamma w, \varphi_{3}\right)_{\omega} \text { for all } \varphi_{3} \in \mathfrak{H}_{3} .
\end{gathered}
$$

If $w \in \mathfrak{H}_{3}$, then, according to the bound $c \rho(x)^{2 \alpha}$ for the moduli of the entries in $\mathcal{A}_{\sharp 3}$, we have $\rho^{-\frac{\alpha}{2}} \mathcal{A}_{\sharp 3} \mathcal{D}_{3}\left(\nabla_{x}\right) w \in L^{2}(\omega)$ and, therefore, the right-hand side of (3.13) implies a continuous functional in $\mathfrak{H}_{\sharp}$. Since $\mathcal{D}_{\sharp}\left(\nabla_{x}\right) \mathcal{R}_{\sharp}=\{0\}$, this functional vanishes on $\mathcal{R}_{\sharp}$, which is required in Theorem 2.10.2. In order to apply this theorem to problem (3.13), one may assume for the time being that the matrix $\mathcal{A}$ takes the diagonal form $\operatorname{diag}\left\{\mathcal{A}_{\sharp \sharp}, \mathcal{A}_{33}\right\}$ and the problem decouples. Hence, the problem (3.13) has a solution $v \in \mathfrak{H}_{\sharp}$ for any $w \in \mathfrak{H}_{3}$ and, fixing somehow a linear inverse operator in the nonuniqueness case $\alpha \geq 1$, we introduce the mapping

$$
w \in \mathfrak{H}_{3} \longmapsto \rho^{\frac{\alpha}{2}} \mathcal{D}_{\sharp}\left(\nabla_{x}\right) v \in L^{2}(\omega) .
$$

Notice that our choice of the inverse does not influence the right-hand side of (3.15) due to the same reason as above. Then the Riesz representation theorem yields the continuous operator

$$
w \in \mathfrak{H}_{3} \longmapsto \mathcal{B} w \in \mathfrak{H}_{3}^{*}
$$

according to the formula

$$
(\mathcal{B} w)\left(\varphi_{3}\right)=\left(\mathcal{A}_{3 \sharp} \mathcal{D}_{\sharp}\left(\nabla_{x}\right) v, \mathcal{D}_{3}\left(\nabla_{x}\right) \varphi_{3}\right)_{\omega} \text { for all } \varphi_{3} \in \mathfrak{H}_{3} .
$$

We now insert (3.17) into (3.14) and obtain

$$
B\left(w, \varphi_{3}\right):=a_{e 3}\left(w, \varphi_{3}\right)+(\mathcal{B} w)\left(\varphi_{3}\right)=\lambda\left(\gamma w, \varphi_{3}\right) \text { for all } \varphi_{3} \in \mathfrak{H}_{3},
$$

where $a_{e 3}\left(w, \varphi_{3}\right)$ is the first scalar product on the left of (3.14).

Lemma 3.3. The quadratic form

$$
\left\langle w, \varphi_{3}\right\rangle_{3}:=B\left(w, \varphi_{3}\right)+\left(\gamma w, \varphi_{3}\right)_{\omega}
$$

defines a scalar product in the Hilbert space $\mathfrak{H}_{3}$.

Proof. By the definition of the norm (2.7) and the formula (1.30) it suffices to verify that

$$
\left\|\rho^{\frac{3 \alpha}{2}} \nabla_{x}^{2} w\right\|_{L^{2}(\omega)} \leq C B(w, w) \text { for all } w \in \mathfrak{H}_{3} .
$$

Due to (3.17) and (3.16) we have

$$
B(w, w)=\left(\mathcal{A}_{33} \mathcal{D}_{3}\left(\nabla_{x}\right) w, \mathcal{D}_{3}\left(\nabla_{x}\right) w\right)_{\omega}+\left(\mathcal{A}_{3 \sharp} \mathcal{D}_{\sharp}\left(\nabla_{x}\right) v, \mathcal{D}_{3}\left(\nabla_{x}\right) w\right)_{\omega},
$$

where $v \in \mathfrak{H}_{\sharp}$ is a solution of the problem (3.13) and, therefore,

$$
\left(\mathcal{A}_{\sharp \sharp} \mathcal{D}_{\sharp}\left(\nabla_{x}\right) v, \mathcal{D}_{\sharp}\left(\nabla_{x}\right) v\right)_{\omega}+\left(\mathcal{A}_{\sharp 3} \mathcal{D}_{3}\left(\nabla_{x}\right) w, \mathcal{D}_{\sharp}\left(\nabla_{x}\right) v\right)_{\omega}=0 .
$$

Summing up (3.21) and (3.22), we see that there exists $C_{\mathcal{A}}>0$ such that

$$
B(w, w)=\left(\mathcal{A D}\left(\nabla_{x}\right) u, \mathcal{D}\left(\nabla_{x}\right) u\right)_{\omega} \geq C_{\mathcal{A}}\|u ; \mathfrak{H}\|^{2} \geq C_{\mathcal{A}}\left\|w ; \mathfrak{H}_{3}\right\|^{2} \text { for all } w \in \mathfrak{H}_{3},
$$

where $u=\left(v_{1}, v_{2}, w\right)^{\top}$ and where we used the inequality (2.42). 
It suffices to verify that the form (3.19) is symmetric. We write

$$
\begin{aligned}
& \left\langle w, \varphi_{3}\right\rangle_{3}=\left(\mathcal{A}_{33} \mathcal{D}_{3}\left(\nabla_{x}\right) w, \mathcal{D}_{3}\left(\nabla_{x}\right) \varphi_{3}\right)_{\omega}+\left(\mathcal{A}_{3 \sharp} \mathcal{D}_{\sharp}\left(\nabla_{x}\right) v, \mathcal{D}_{3}\left(\nabla_{x}\right) \varphi_{3}\right)_{\omega}, \\
& \left\langle\varphi_{3}, w\right\rangle_{3}=\left(\mathcal{A}_{33} \mathcal{D}_{3}\left(\nabla_{x}\right) \varphi_{3}, \mathcal{D}_{3}\left(\nabla_{x}\right) w\right)_{\omega}+\left(\mathcal{A}_{3 \sharp} \mathcal{D}_{\sharp}\left(\nabla_{x}\right) \psi, \mathcal{D}_{3}\left(\nabla_{x}\right) w\right)_{\omega},
\end{aligned}
$$

where $\psi \in \mathfrak{H}_{\sharp}$ is a solution of the problem (3.13) with $w$ replaced by $\varphi_{3}$. Taking $v$ as a test function in this problem, we have

$$
\left(\mathcal{A}_{\sharp \sharp} \mathcal{D}_{\sharp}\left(\nabla_{x}\right) \psi, \mathcal{D}_{\sharp}\left(\nabla_{x}\right) v\right)_{\omega}=-\left(\mathcal{A}_{\sharp 3} \mathcal{D}_{3}\left(\nabla_{x}\right) \varphi_{3}, \mathcal{D}_{\sharp}\left(\nabla_{x}\right) v\right)_{\omega} .
$$

Hence, recalling the symmetry of the matrix (1.24), we derive from (3.23) that

$$
\left\langle w, \varphi_{3}\right\rangle_{3}=\left(\mathcal{A}_{33} \mathcal{D}_{3}\left(\nabla_{x}\right) w, \mathcal{D}_{3}\left(\nabla_{x}\right) \varphi_{3}\right)_{\omega}-\left(\mathcal{A}_{\sharp \sharp} \mathcal{D}_{\sharp}\left(\nabla_{x}\right) v, \mathcal{D}_{\sharp}\left(\nabla_{x}\right) \psi\right)_{\omega} .
$$

A similar argument turns (3.24) into

$$
\left\langle\varphi_{3}, w\right\rangle_{3}=\left(\mathcal{A}_{33} \mathcal{D}_{3}\left(\nabla_{x}\right) \varphi_{3}, \mathcal{D}_{3}\left(\nabla_{x}\right) w\right)_{\omega}-\left(\mathcal{A}_{\sharp \sharp} \mathcal{D}_{\sharp}\left(\nabla_{x}\right) \psi, \mathcal{D}_{\sharp}\left(\nabla_{x}\right) v\right)_{\omega} .
$$

All the desired properties of the form (3.19) have now been checked.

3.4. The spectrum for bending the plate. We now introduce the new spectral parameter

$$
\tau=(1+\lambda)^{-1}
$$

and the operator $T_{3}$ in $\mathfrak{H}_{3}$ by the formula

$$
\left\langle T_{3} w, \varphi_{3}\right\rangle_{3}=\left(\gamma w, \varphi_{3}\right)_{\omega}, \quad w, \varphi_{3} \in \mathfrak{H}_{3} .
$$

In this way, problem (3.18), and therefore (3.13), turns into the abstract equation

$$
T_{3} w=\tau w \text { in } \mathfrak{H}_{3} .
$$

The operator $T_{3}$ is positive, symmetric, and continuous, therefore self-adjoint. In the case $\alpha<2$ the imbedding $\mathfrak{H}_{3} \subset \mathcal{L}$ is compact (see section 3.2) and, hence, the spectrum of $T_{3}$ is discrete, except for the point $\tau=0$, which implies an essential spectrum (see [2, Thm. 10.1.5]). The formula (3.25) passes the discreteness property to the spectrum of the problem (3.13) and then to the spectrum of the problem (3.12).

Let us prove that in the case $\alpha \geq 2$ the essential spectrum of $T_{3}$ does not consist of the only point $\tau=0$. In view of [2, Thm. 10.1.5] it is sufficient to verify that the imbedding $\mathfrak{H}_{3} \subset \mathcal{L}$ is not compact.

We introduce the cut-off function $\chi_{m}(x)$ which vanishes outside $\omega_{d}=\{x \in \omega$ : $\operatorname{dist}(x, \partial \omega)<d\}$ and is given inside $\omega_{d}$ by

$$
\chi_{m}(x)=\chi\left(\frac{2^{4 m+3} x}{d}\right)\left(1-\chi\left(\frac{2^{4 m} x}{d}\right)\right),
$$

where $m \in \mathbb{N}$ and $\chi$ is the standard cut-off function (2.32). The function $\chi_{m}$, with its graph drawn in Figure 3, satisfies

$$
\chi_{m}(x)= \begin{cases}0 & \text { for } \quad x \geq 2^{-4 m} d \text { and } x \leq 2^{-4 m-4} d, \\ 1 & \text { for } \quad 2^{-4 m-3} d \leq x \leq 2^{-4 m-1} d,\end{cases}
$$

Copyright $@$ by SIAM. Unauthorized reproduction of this article is prohibited. 


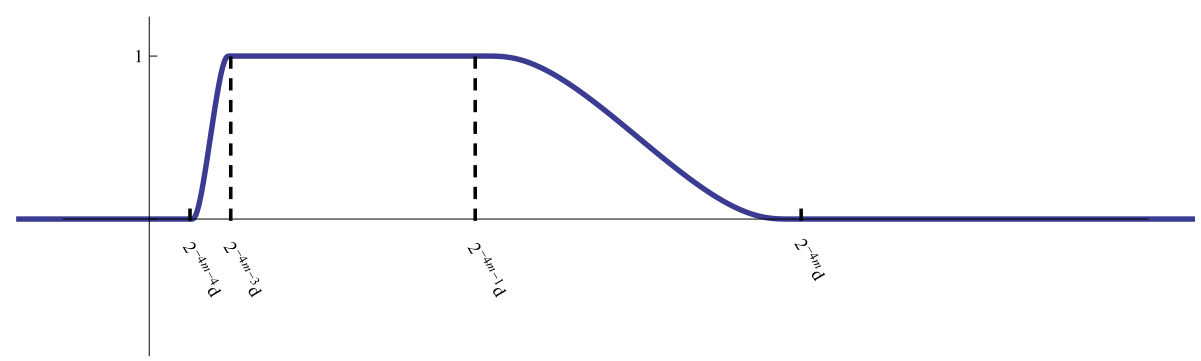

FIG. 3. The cut-off function $\chi_{m}$ from (3.28).

so that, if $p \neq m$,

$$
\chi_{m}(x) \chi_{p}(x)=0 \text { for all } x \in \omega .
$$

With any smooth function $z$ on $\partial \omega$, we set

$$
z_{m}(s)=z(s) \chi_{m}(s) .
$$

Since $\chi_{m} \in C_{c}^{\infty}(\omega)$, the function (3.31) belongs to $\tilde{H}_{3}$. Moreover, in view of (3.29) we obtain

$$
\left\|z_{m} ; \mathcal{L}\right\|^{2} \geq c \int_{\partial \omega}|z(s)|^{2} \int_{2^{-4 m-3}}^{2^{-4 m-1}} \nu^{\alpha} d \nu d s \geq c_{z}^{0} 2^{-4 m(\alpha+1)}
$$

for some $c_{z}^{0}>0$. Similarly,

$$
\left\|z_{m} ; \mathcal{L}\right\|^{2} \leq C_{z}^{0} 2^{-4 m(\alpha+1)} .
$$

Finally, we calculate

$$
\left\|\rho^{\frac{3 \alpha}{2}} \nabla_{x}^{2} z_{m}\right\|_{L^{2}(\omega)}^{2} \leq C_{z}^{1} \int_{\partial \omega}\left(2^{-4 m}\right)^{4} \int_{2^{-4 m-3}}^{2^{-4 m-1}} \nu^{3 \alpha} d \nu d s \leq C_{z}^{1} 2^{-4 m(-4+3 \alpha+1)}
$$

since $3 \alpha-3 \geq \alpha+1$ for $\alpha \geq 2$, the sequence $\left(Z_{m}\right)$ of functions $Z_{m}=2^{2 m(\alpha+1)} z_{m}$ is bounded in $\mathfrak{H}_{3}$, hence, a subsequence converges weakly in $\mathfrak{H}_{3}$ and the limit is zero due to (3.30). However, no subsequence can converge strongly in $\mathcal{L}$ to 0 since $\left\|Z_{m} ; \mathcal{L}\right\| \geq \sqrt{C_{Z}^{0}}>0$. Thus the imbedding $\mathfrak{H}_{3} \subset \mathcal{L}$ is not compact and the essential spectrum of $T_{3}$ is bigger than just the point $\tau=0$. This means that, by (3.25), the essential spectrum of the problem (3.18) is not empty. Due to the reduction of (3.12) to (3.18) made above, this spectral property is attributed to the system (1.29) describing transversal oscillations of the plate and to its weak formulation in (3.12).

THEOREM 3.4. If $\alpha<2$, the spectrum of the problem (3.18), which, by definition, implies the spectrum of the problem (3.12), is fully discrete. However, for $\alpha>2$ the essential spectrum of (3.18) is not empty.

Remark 3.5. The authors are not yet able to find a point of the essential spectrum in the case $\alpha=2$.

Proof. For $\alpha<2$ the proof is given in the arguments leading to the theorem.

In order to show that the essential spectrum is nonempty in the case $\alpha>2$, it is sufficient to construct a singular Weyl sequence. If $\alpha>2$, a subsequence in $\left(Z_{m}\right)$, still denoted by $\left(Z_{m}\right)$, keeps the properties

$$
\begin{aligned}
& \text { (i) }\left\|Z_{m} ; \mathfrak{H}_{3}\right\| \geq c>0, \\
& \text { (ii) } Z_{m} \rightarrow 0 \text { weakly in } \mathfrak{H}_{3}, \\
& \text { (iii) }\left\|T_{3} Z_{m}-Z_{m} ; \mathfrak{H}_{3}\right\| \rightarrow 0
\end{aligned}
$$

Copyright $@$ by SIAM. Unauthorized reproduction of this article is prohibited. 
of the singular Weyl sequence for operator $T_{3}$ at the point $\tau=1$. By the Weyl criterion (see, e.g., [2, Thm. 9.1.2], the point $\tau=1$ belongs to the essential spectrum of $T_{3}$ and, therefore, the point $\lambda=1-\tau^{-1}=0$ belongs to the essential spectrum of the problem (3.18).

The properties (i) and (ii) follow from (3.28), (3.32)-(3.34). To confirm the third one, we use the definitions (3.26), (3.19), and observe that

$$
\begin{gathered}
\left\|T_{3} Z_{m}-Z_{m} ; \mathfrak{H}_{3}\right\|=\sup \left\{\left\langle T_{3} Z_{m}-Z_{m}, W\right\rangle ; W \in \mathfrak{H}_{3} \text { with }\left\|W ; \mathfrak{H}_{3}\right\|=1\right\} \\
=\sup \left\{\left(\gamma Z_{m}, W\right)_{\omega}-B\left(Z_{m}, W\right)-\left(Z_{m}, W\right)_{\omega} ; W \in \mathfrak{H}_{3} \text { with }\left\|W ; \mathfrak{H}_{3}\right\|=1\right\} \\
=\sup \left\{B\left(Z_{m}, W\right) ; W \in \mathfrak{H}_{3} \text { with }\left\|W ; \mathfrak{H}_{3}\right\|=1\right\} .
\end{gathered}
$$

Hence

$$
\left\|T_{3} Z_{m}-Z_{m} ; \mathfrak{H}_{3}\right\| \leq\left\|\rho^{\frac{3 \alpha}{2}} \nabla_{x}^{2} Z_{m}\right\|_{L^{2}(\omega)} \leq c 2^{2 m(\alpha+1)} 2^{-2 m(-4+3 \alpha+1)}=c 2^{2 m(4-2 \alpha)} .
$$

For $\alpha>2$, the last bound goes to zero as $m \rightarrow+\infty$. Thus, all three properties hold true.

\section{Final remarks.}

4.1. Continuous spectra in elasticity. In $[30,32,33,1]$ and $[3,4]$ it was proved that peak - and beak - shaped elastic bodies (see Figure 4 top and right) get both continuous and essential spectra whenever the sharpness exponent satisfies $\alpha \geq 2$. While the corresponding quasi-modes, leading to singular Weyl sequences, are related to the bending oscillations of the thinning irregularities of the surfaces. Those results are in perfect correlation with the information on the spectra obtained in section 3. Problem (3.3) on the longitudinal oscillations has the discrete spectrum but the problems (3.10) and (3.12), involving the transversal oscillation of the plate, display essential spectra under the same condition $\alpha \geq 2$. However, spectra of elastic bodies with cuspidal edges (cf. Figure 1 and Figure 4 (bottom)) are not rigorously investigated yet.

The essential spectrum always coincides with the continuous spectrum in peakshaped domains (see [33]), but this fact is neither proved nor disproved yet for beakshaped solids as well as for the problem (1.29). In other words, there is no result on the existence of eigenvalues with infinite multiplicity.

The complete description of the structure of the spectra is not given yet for the above-mentioned elasticity problems with the one exception. In [1] it is verified that the continuous spectrum implies the closed positive real semi-axis in the case of a supersharp peak meaning $\alpha>2$. See Figure 4 (top).

4.2. Asymptotic analysis. The formal asymptotic procedure of dimension reduction, described, e.g., in [38, 6, 35, 37] and [28, Chap. 4], works directly for thin $3 \mathrm{~d}$ plates with sharp edges (Figure 1) and/or degenerating thickness (Figure 5) as well. The justification of the main terms in the formal asymptotics of the elastic fields in the plate ought to be based on specific weighted inequalities of Korn's type. An approach to derive such inequalities can be taken from [23, 32] but needs a slight modification. The authors know only one paper [29] where all of these steps were realized, namely in the Neumann problem for a scalar elliptic second order equation in the domain drawn in Figure 1.

The clamped edge of the thin plate (1.20) assumes that a small part of the plate surface (cf. Figure 6 and Figure 1) is fixed by means of the Dirichlet conditions for 

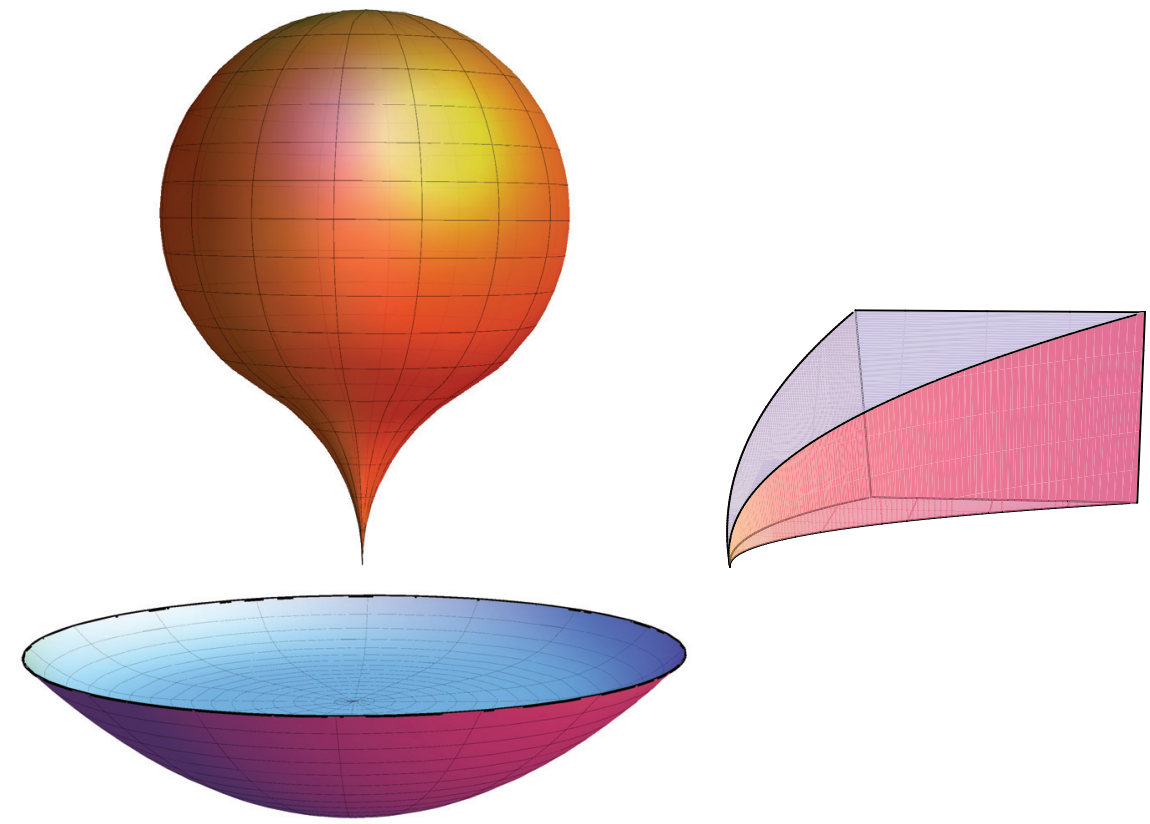

FIG. 4. Elastic bodies with a singularity: a peak shaped (top), a beak shaped (right), and another one with a cuspidal edge.

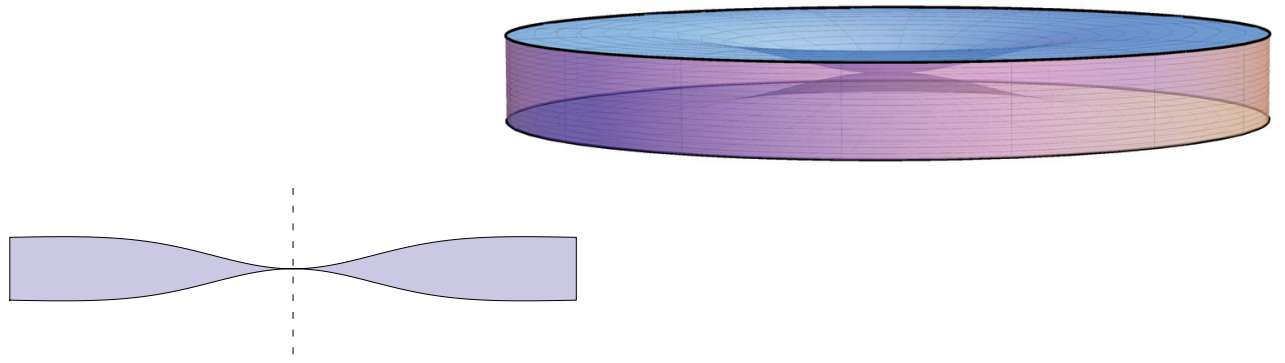

FIG. 5. On the right, a plate with a thin middle section; on the left, a cross section.

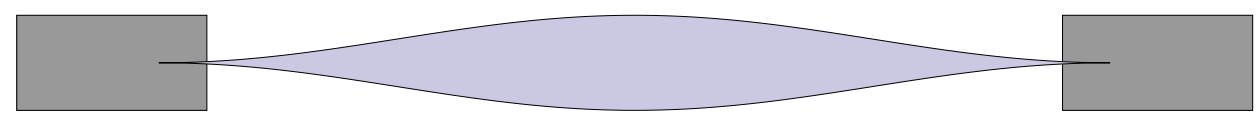

FIG. 6. A clamped plate with a sharp edge.

the 3-d displacement field. In the vicinity of the collision line of the Dirichlet and Neumann (traction-free) conditions, the boundary layer phenomenon occurs. Such a boundary layer however is never dealt with, not even in the scalar case [29].

The general theory of exponential boundary layers in thin cylindrical domains was developed in [22] (see also [17, Chap. 15, 16]) and applied to cylindrical elastic plates in $[39,24,25,7,8,9]$ and others. However, in a thin domain with this sharp edge the general procedure must be modified crucially and, in particular, the boundary layers 
get only a power-law decay (cf. [29] for a scalar equation). Moreover, in the case of the traction-free edge the formulation of any boundary condition for the systems (1.17), (1.29), and (1.31) is not clear at all. It would be very interesting to find out whether the Mikhlin result on the discrete spectrum and our result on the continuous spectrum are still valid.

\section{REFERENCES}

[1] F. L. Bakharev and S. A. Nazarov, On the structure of the spectrum of a problem in the elasticity for a body with a supersharp spike, Sibirsk. Mat. Zh., 50 (2009), pp. 746-756 (in Russian); Sib. Math. J., 50 (2009), pp. 587-595 (in English).

[2] M. Sh. Birman and M. Z. Solomjak, Spectral theory of selfadjoint operators in Hilbert space, translated from the 1980 Russian original by S. Khrushchëv and V. Peller, Math. Appl. (Soviet Ser.), D. Reidel Publishing Co., Dordrecht, The Netherlands, 1987.

[3] G. Cardone, S. A. Nazarov, and J. Taskinen, The "absorption" effect for elastic waves caused by a beak-shaped boundary irregularity, Dokl. Akad. Nauk, 425 (2009), pp. 182-186 (in Russian).

[4] G. Cardone, S. A. Nazarov, and J. Taskinen, A criterion for the existence of the essential spectrum for beak-shaped elastic bodies, J. Math. Pures Appl. (9), 92 (2009), pp. 628-650.

[5] P. G. Ciarlet And S. Kesavan, Two-dimensional approximations of three-dimensional eigenvalue problems in plate theory, Comput. Methods Appl. Mech. Engrg., 26 (1981), pp. 145172.

[6] P. G. Ciarlet, Plates and Junctions in Elastic Multi-Structures. An Asymptotic Analysis, Rech. Math. Appl., 14, Masson, Paris; Springer-Verlag, Berlin, 1990.

[7] M. Dauge and I. Gruais, Edge layers in thin elastic plates, in The Seventh Conference on Numerical Methods and Computational Mechanics in Science and Engineering (NMCM 96), (Miskolc). Comput. Methods Appl. Mech. Engrg., 157 (1998), pp. 335-347.

[8] M. Dauge, I. Duurdjevic, E. Faou, And A. Rössle, Eigenmode asymptotics in thin elastic plates, J. Math. Pures Appl. (9), 78 (1999), pp. 925-964.

[9] M. Dauge, I. Gruais, and A. Rössle, The influence of lateral boundary conditions on the asymptotics in thin elastic plates, SIAM J. Math. Anal., 31 (1999), pp. 305-345.

[10] G. H. Hardy, J. E. Littlewood, and G. Pólya, Inequalities, 2nd ed, Cambridge University Press, Cambridge, UK, 1952.

[11] V. A. Kondratı̌ev and O. A. Oleinik, Boundary value problems for the system of elasticity theory in unbounded domains. Korns inequalities, Uspehi Mat. Nauk., 43 (1988), pp. 55-98 (in Russian); Russian Math. Surveys, 43 (1988), pp. 65-119 (in English).

[12] V. V. Krylov, New type of vibration dampers utilising the effect of acoustic "black holes," Acta Acustica united with Acustica, 90 (2004), pp. 830-837.

[13] A. Kufner and L.-E. Persson, Weighted Inequalities of Hardy Type, World Scientific, River Edge, NJ, 2003.

[14] O. A. Ladyzhenskaya, The Boundary Value Problems of Mathematical Physics, Appl. Math. Sci., 49, Springer-Verlag, New York, 1985.

[15] S. G. Lekhnitskĭ, Theory of Elasticity of an Anisotropic Body, translated from the second Russian edition, Mir, Moscow, 1981.

[16] E. V. Makhover, On the spectrum of eigenvalues of a plate with sharp edge, Sci. Notes of Leningrad Pedagogical Gerzen Institute, 197 (1958), pp. 113-118 (in Russian).

[17] W. G. Mazja, S. A. Nasarow, B. A. Plamenewski, Asymptotische Theorie elliptischer Randwertaufgaben in singulär gestörten Gebieten. 2, Berlin: Akademie-Verlag, Berlin, 1991 (translation in V. Maz'ya, S. Nazarov, and B. Plamenevskij, Asymptotic theory of elliptic boundary value problems in singularly perturbed domains, Vol. 2, Birkhäuser Verlag, Basel, 2000).

[18] S. G. Mikhlin, Variational methods in mathematical physics, translated by T. Boddington, The Macmillan Co., New York, 1964.

[19] S. G. Mikhlin, Variational Methods in Mathematical Physics, 2nd ed., revised and augmented, Izdat. Nauka, Moscow, 1970 (in Russian).

[20] M. A. Mironov, Propagation of a flexural wave in a plate whose thickness decreases smoothly to zero in a finite interval, Soviet Physics: Acoustics, 34 (1988), pp. 318-319.

[21] O. V. Motygin and S. A. NAZARov, Justification of the Kirchhoff hypotheses and error estimation for two-dimensional models of anisotropic and inhomogeneous plates, including laminated plates, IMA J. Appl. Math., 65 (2000), pp. 1-28. 
[22] S. A. NAZARov, The structure of solutions of elliptic boundary value problems in slender domains, Vestnik Leningrad Univ., 15 (1982), pp. 65-68 (in Russian); Vestn. Leningr. Univ. Math., 15 (1983), pp. 99-104 (in English).

[23] S. A. Nazarov, Weighted Korn inequalities in parabolic domains, Mat. Zametki., 62 (1997), pp. 751-765 (in Russian); correction in Mat. Zametki., 63 (1998), p. 640, Math. Notes, 62 (1997), pp. 629-641 (in English); erratum in Math. Notes, 52 (1998), p. 565 (in English).

[24] S. A. NAZAROV, On the accuracy of asymptotic approximations for longitudinal deformation of a thin plate, RAIRO Modél. Math. Anal. Numér., 30 (1996), pp. 185-213.

[25] S. A. NAZArov, Boundary layers and boundary conditions for hinge support for thin plates, Zap. Nauchn. Sem. S.-Peterburg. Otdel. Mat. Inst. Steklov. (POMI), 257 (1999), Mat. Vopr. Teor. Rasprostr. Voln. 28, pp. 228-287, 351-352 (in Russian); J. Math. Sci. (New York), 108 (2002), pp. 806-850 (in English).

[26] S. A. NAZARov, On the asymptotics of the spectrum of a problem in elasticity theory for a thin plate, Sibirsk. Mat. Zh., 41 (2000), pp. 895-912 (in Russian); Siberian Math. J., 41 (2000), pp. 744-759 (in English).

[27] S. A. NAZARov, Estimating the convergence rate for eigenfrequencies of anisotropic plates with variable thickness, C. R. Mecanique, 330 (2002), pp. 603-607.

[28] S. A. Nazarov, Asymptotic Theory of Thin Plates and Rods. Vol. 1. Dimension Reduction and Integral Estimates, Nauchnaya Kniga, Novosibirsk, 2002 (in Russian).

[29] S. A. NazArov AND J. TASkinen, Asymptotics of a solution to the Neumann problem in a thin domain with the sharp edge, Zap. Nauchn. Sem. St.-Petersburg Otdel. Mat. Inst. Steklov, 332 (2006), pp. 193-219 (in Russian); Journal of Math. Sci., 142 (2007), pp. 2630-2644 (in English).

[30] S. A. NAzARov, A criterion for the continuous spectrum for elasticity and other self-adjoint systems on sharp peak-shaped domains, C.R. Mecanique, 335 (2007), pp. 751-756.

[31] S. A. Nazarov, Korn's inequalities for elastic joints of massive bodies, thin plates, and rods, Uspekhi Mat. Nauk, 63 (2008), pp. 37-110 (in Russian); Russian Math. Surveys, 63 (2008), pp. 35-107 (in English).

[32] S. A. NAZARov, On the spectrum of a problem in the theory of elasticity for a spiked body, Sibirsk. Mat. Zh., 49 (2008), pp. 1105-1127 (in Russian); Sib. Math. J., 49 (2008), pp. 874893 (in English).

[33] S. A. NAZARov, On the essential spectrum of boundary value problems for systems of differential equations in a bounded domain with a cusp, Funktsional. Anal. i Prilozhen, 43 (2009), pp. 55-67 (in Russian); Funct. Anal. Appl. 43, (2009), pp. 44-54 (in English).

[34] B. OpIC And A. Kufner, Hardy-type inequalities, in Pitman Res. Notes in Math., 219, Longman Scientific \& Technical, Harlow; 1990.

[35] G. Panasenko, Multi-Scale Modelling for Structures and Composites, Springer, Dordrecht, The Netherlands, 2005.

[36] F. Riesz And B. Sz.-NAGY, Functional Analysis reprint of the 1955 original, Dover Publications, Inc., New York, 1990.

[37] J. Sanchez Hubert and E. Sanchez-Palencia, Coques Élastiques Minces, Propriétés Asymptotiques, Masson, Paris, 1997.

[38] B. A. ShOIKHET, On asymptotically exact equations of thin plates of complex structure, J. Appl. Math. Mech., 37 (1973) pp. 867-877 (in Russian); (translated from Prikl. Mat. Meh., 37 (1973), pp. 914-924 (in English).

[39] I. S. Zorin AND S. A. NAZAROV, Edge effect in the bending of a thin three-dimensional plate, Prikl. Mat. Mekh., 53 (1989), pp. 642-650 (in Russian); J. Appl. Math. Mech., 53 (1989), pp. 500-507 (in English).

Copyright (c) by SIAM. Unauthorized reproduction of this article is prohibited. 